\title{
Set-theoretic Methods in Robust Detection and Isolation of Sensor Faults
}

\author{
Feng Xu ${ }^{a, *}$, Vicenç Puig $^{a}$, Carlos Ocampo-Martínez $^{a}$, Sorin Olaru ${ }^{b}$ and Florin Stoican ${ }^{c}$ \\ ${ }^{a}$ Institut de Robòtica i Informàtica Industrial (CSIC-UPC), Technical University of Catalonia (UPC) \\ Llorens i Artigas, 4-6, 2nd floor, 08028, Barcelona, Spain \\ ${ }^{b}$ E3S (SUPELEC Systems Sciences), Automatic Control Department, Gif sur Yvette, France \\ ${ }^{c}$ Department of Automatic Control and Systems Engineering, Faculty of Automatic Control and Computers \\ "Politehnica" University of Bucharest, 313 Spl. Independentei, 060042 Bucharest, Romania
}

\begin{abstract}
This paper proposes a sensor-fault detection and isolation (FDI) approach based on interval observers and invariant sets. In fault detection (FD), both interval observer-based and invariant set-based mechanisms are used to provide real-time fault alarms. In fault isolation (FI), the proposed approach also uses these two different mechanisms. The former, based on interval observers, aims to isolate faults during the transient-state operation induced by faults. If the former does not succeed, the latter, based on both interval observers and invariant sets, is started to guarantee FI after the system enters into steady state. Besides, a collection of invariant set-based FDI conditions are established by using all available system-operating information provided by all interval observers. In order to reduce computational complexity, a method to remove all available but redundant/unnecessary system-operating information is incorporated into this approach. If the considered faults satisfy the proposed FDI conditions, it can be guaranteed that they are detectable and isolable after their occurrences. This paper concludes with a case study based on a subsystem of a wind turbine benchmark, which can illustrate the effectiveness of this FDI technique.
\end{abstract}

Keywords: Fault detection and isolation, interval observers, positively invariant sets, linear systems, zonotopes.

\section{Introduction}

As the industry advances, the technical systems become more and more sophisticated. Generally, the more complex the system is, the more likely it is affected by faults. In order to protect the system once it becomes faulty, it is necessary to timely detect and isolate faults and take remedial measures, which requires effective FDI techniques (Blanke et al. 2006). This paper focuses on the set-theoretic methods for detection and isolation of sensor faults (Blanchini and Miani 2008). In the literature, the set-theoretic fault diagnosis, known as a class of robust techniques, is mainly involved in set-membership estimation, interval observers and invariant sets (Blesa et al. 2011; Puig 2010; Seron et al. 2012).

Set-membership estimation and interval observers provide set-valued estimations for both states and outputs of a monitored system in real time. The FD principles of both techniques consist in testing the consistency between the measured outputs and their corresponding estimated sets. If the measured outputs are consistent with the estimated output sets, it is considered that the system is healthy, otherwise it implies that the system has become faulty. In the literature, set-membership estimation is used for FD and rarely for FI (Chai and Qiao 2014), while interval observers have been extended for FI mainly in terms of actuator faults in the previous works of the authors (Xu et al. 2013, 2014).

Instead of estimating state and output sets on-line, the invariant set-based approach relies on the invariant set characterization of system behaviors at steady state (Kofman et al. 2007). It is known that a

${ }^{*}$ Corresponding author. e-mail: fxu@iri.upc.edu 
system always has several different modes, a healthy one and at least one faulty one. For each mode, the corresponding invariant set to confine the residual (a signal sensitive to faults) can be constructed. If the invariant sets of all modes are separate from each other, FD can be implemented by testing whether the residual goes out of its healthy invariant set. After FD, the fault can be isolated by searching which faulty invariant set the residual finally converges to (Seron et al. 2008, 2012).

Within the knowledge range of the authors, a brief summary of the set-theoretic methods related to this paper is presented. Set-membership state estimation based on zonotopes was discussed in (Alamo et al. 2005; Combastel 2003; Le et al. 2013). The application of set-membership estimation to fault diagnosis of a wind turbine benchmark can be found in (Blesa et al. 2011), and interval observers are used for robust state estimation in (Mazenc and Bernard 2011), where the readers are referred to. In (Puig et al. 2003; Guerra et al. 2008; Raïssi et al. 2010), FD techniques based on interval observers are proposed. In (Seron et al. 2008; Olaru et al. 2010), invariant sets are used to implement sensor FDI in a multisensor fault-tolerant control (FTC) scheme.

This paper has two main objectives. The first one is to summarize and collect the previous works of the authors (Xu et al. 2013, 2014) and make the picture more clear and systematic. The second one is to propose a set-based robust sensor FDI approach with no need of multisensor redundancy. It is stated that, to improve the set-based FDI approach, one has to propose new techniques with tight guaranteed FDI conditions and less complexity. Because of different characteristics between actuator and sensor faults, one has to pay more efforts to implement set-based robust detection and isolation for sensor faults.

The contribution of this paper is threefold. First, the proposed sensor FDI approach combines the advantages of interval observers in the transient-state regime with the advantages of invariant sets describing the steady-state system operation. Second, the FDI decision is jointly made by both interval observer-based and invariant set-based FDI mechanisms. Third, the proposed approach uses the available system-operating information from all interval observers, selects all useful information for FDI conditions and FDI implementation but discards all available but unnecessary/redundant system-operating information to reduce computational complexity. These mentioned points imply to obtain a balance between computational complexity and FDI conservatism.

In the remaining of this paper, Section II introduces the proposed FDI scheme. Section III defines the residual signal and constructs relevant invariant sets. Interval observer-based and invariant set-based FDI mechanisms are briefly recapitulated in Section IV. In Section V, FDI conditions for the proposed technique are given. An FDI algorithm is used to summarize this approach in Section VI. In Section VII, a case study based on a subsystem of a wind turbine benchmark is used to illustrate the effectiveness of the FDI approach. Finally, Section VIII draws the general conclusions.

Throughout this paper, the notation $\mathbb{B}^{r}$ denotes a box composed of $r$ unitary intervals, $\mathbb{R}^{+}$denotes the set of positively real numbers, $\mathbb{R}^{m \times n}$ represents the $m \times n$ real matrix space, the inequalities are understood elementwise, the symbol $\oplus$ denotes the Minkowski sum, $I$ denotes the identity matrix with suitable dimensions, $a^{T}$ denotes the transpose of a matrix or vector $a,[\bar{x}, \underline{x}]$ denotes an interval, $\left(\left[\bar{x}_{1}, \underline{x}_{1}\right],\left[\bar{x}_{2}, \underline{x}_{2}\right], \ldots,\left[\bar{x}_{n}, \underline{x}_{n}\right]\right)^{T}$ denotes an $n$-dimensional interval vector, diag $(\cdot)$ denotes the diagonal matrix and $\|\cdot\|_{s}$ is the Euclidean vector $s$-norm.

\section{Problem Formulation}

\subsection{Plant Models}

The FDI scheme in Figure 1 assumes that the plant under the effect of sensor faults is modelled as

$$
\begin{aligned}
x_{k+1} & =A x_{k}+B u_{k}+\omega_{k}, \\
y_{k} & =G_{i} C x_{k}+\eta_{k},
\end{aligned}
$$

where $A \in \mathbb{R}^{n \times n}, B \in \mathbb{R}^{n \times p}$ and $C \in \mathbb{R}^{q \times n}$ are time-invariant matrices, $x_{k} \in \mathbb{R}^{n}, u_{k} \in \mathbb{R}^{p}$ and $y_{k} \in \mathbb{R}^{q}$ are states, inputs and outputs at time instant $k$, respectively, and $\omega_{k}$ and $\eta_{k}$ are process disturbances and 
measurement noises, respectively.

In (1), $G_{i}(i \in \mathcal{I}=\{0,1, \ldots, N\})$ is a $q \times q$ diagonal matrix which characterizes the sensor functioning modes. The diagonal elements take values from an interval [0,1] (i.e., the $i$-th diagonal value characterizes the status of the corresponding sensor with 0 describing a total outage, a value inside $(0,1)$ a partial outage and 1 healthy functioning, respectively). By convention, one assumes $G_{0}$ to be the identity matrix (denoting the healthy sensor mode) and $G_{i}$ with $i \neq 0$ a priori known faulty modes.

Remark 2.1. The number $N$ of the considered faulty modes is different from that of the sensors in a system, because there may be several fault modes corresponding to one sensor or one fault mode corresponding to several sensors.

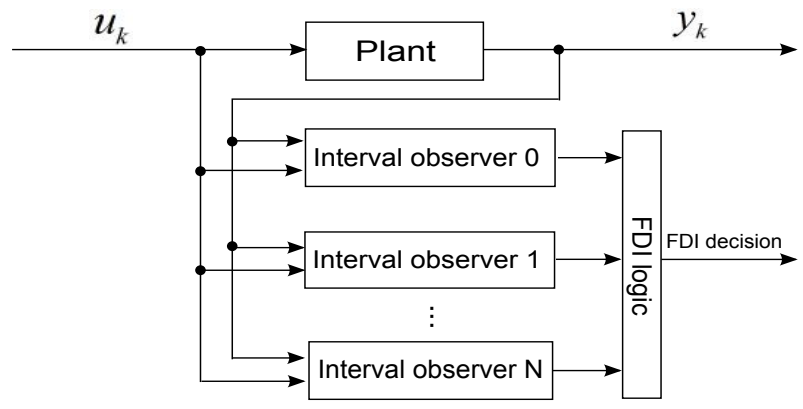

Figure 1. Sensor FDI scheme

Assumption 2.1. The disturbances and noises $\omega_{k}$ and $\eta_{k}$ are bounded by known sets

$$
\begin{aligned}
W & =\left\{\omega \in \mathbb{R}^{n}:\left|\omega-\omega^{c}\right| \leq \bar{\omega}, \omega^{c} \in \mathbb{R}^{n}, \bar{\omega} \in \mathbb{R}^{n}\right\}, \\
V & =\left\{\eta \in \mathbb{R}^{q}:\left|\eta-\eta^{c}\right| \leq \bar{\eta}, \eta^{c} \in \mathbb{R}^{q}, \bar{\eta} \in \mathbb{R}^{q}\right\},
\end{aligned}
$$

respectively, where the vectors $\omega^{c}, \eta^{c}, \bar{\omega}$ and $\bar{\eta}$ have known and constant values.

Remark 2.2. The sets $W$ and $V$ are described by means of zonotopes $W=\omega^{c} \oplus H_{\bar{\omega}} \mathbb{B}^{n}$ and $V=$ $\eta^{c} \oplus H_{\bar{\eta}} \mathbb{B}^{q}$, where $H_{\bar{\omega}} \in \mathbb{R}^{n \times n}$ and $H_{\bar{\eta}} \in \mathbb{R}^{q \times q}$ are diagonal matrices with their main diagonals composed of $\bar{\omega}$ and $\bar{\eta}$, respectively.

Assumption 2.2. The pair $(A, B)$ is stabilizable and the pairs $\left(A, G_{i} C\right)$ are detectable for all $i \in \mathcal{I}$.

Remark 2.3. Considering that the scheme shown in Figure 1 is an open-loop system, the first priority for this scheme is the system stability. Thus, under Assumption 2.2, the input $u_{k}$ should stabilize the system or the system itself is stable (i.e., $A$ is a Schur matrix).

Assumption 2.3. The considered modes can persist sufficiently long time such that the FDI module has enough responsive time to detect and isolate them.

\subsection{Interval Observers}

In Figure 1, a bank of interval observers are designed to monitor the system. In accordance with (1), the interval observer corresponding to the $j$-th sensor mode $(j \in \mathcal{I})$ is designed as

$$
\begin{aligned}
\hat{X}_{k+1}^{j} & =\left(A-L_{j} G_{j} C\right) \hat{X}_{k}^{j} \oplus\left\{B u_{k}\right\} \oplus\left\{L_{j} y_{k}\right\} \oplus\left(-L_{j}\right) V \oplus W, \\
\hat{Y}_{k}^{j} & =G_{j} C \hat{X}_{k}^{j} \oplus V,
\end{aligned}
$$

where $\hat{X}_{k}^{j}$ and $\hat{Y}_{k}^{j}$ are the state and output sets estimated by the $j$-th interval observer, respectively. 
Assumption 2.4. The interval observer gain $L_{j}$ is chosen to assure that $A-L_{j} G_{j} C$ is a Schur matrix (always possible under Assumption 2.2).

Assumption 2.5. The initial state of the plant and the initial state set of all interval observers are denoted as $x_{0}$ and $\hat{X}_{0}$, respectively, and $x_{0} \in \hat{X}_{0}$ holds.

Note that, for different interval observers, the initial sets could be different. However, without loss of generality, one uses one initial set $\hat{X}_{0}$ for all interval observers. Since $W$ and $V$ are zonotopes, if the initial set $\hat{X}_{0}$ is chosen as a zonotope, $\hat{X}_{k+1}^{j}$ and $\hat{Y}_{k}^{j}$ are also zonotopes as the Minkowski sum of zonotopes is a closed operation. By using zonotope operations introduced in Appendices, (3) can be transformed into the equivalent center-segment matrix form

$$
\begin{aligned}
\hat{x}_{k+1}^{j, c} & =\left(A-L_{j} G_{j} C\right) \hat{x}_{k}^{j, c}+B u_{k}+L_{j} y_{k}-L_{j} \eta^{c}+w^{c}, \\
\hat{H}_{k+1}^{j, x} & =\left[\left(A-L_{j} G_{j} C\right) \hat{H}_{k}^{j, x}-L_{j} H_{\bar{\eta}} H_{\bar{\omega}}\right], \\
\hat{y}_{k}^{j, c} & =G_{j} C \hat{x}_{k}^{j, c}+\eta^{c}, \\
\hat{H}_{k}^{j, y} & =\left[G_{j} C \hat{H}_{k}^{j, x} H_{\bar{\eta}}\right],
\end{aligned}
$$

where $\hat{x}_{k}^{j, c}, \hat{y}_{k}^{j, c}, \hat{H}_{k}^{j, x}$ and $\hat{H}_{k}^{j, y}$ are the centers and segment matrices of $\hat{X}_{k}^{j}$ and $\hat{Y}_{k}^{j}$, respectively.

Remark 2.4. Under Assumptions 2.2, 2.4 and 2.5, the state $x_{k}$ is always contained inside the state set estimated by the interval observer matching the current mode if there is no mode switching. The same results hold for the outputs and their corresponding set (i.e., $x_{k} \in \hat{X}_{k}^{j}$ and $y_{k} \in \hat{Y}_{k}^{j}$ ).

\section{Residual Analysis}

In this section, residuals are defined in terms of zonotopes and the corresponding bounding sets of residual zonotopes (named residual-bounding zonotopes) are derived.

\subsection{Residual Definition}

If the system currently operates in the $i$-th mode, the residual zonotope corresponding to the $j$-th interval observer at time instant $k$ is defined as

$$
\begin{aligned}
R_{k}^{i j} & =\left\{y_{k}\right\} \oplus\left(-\hat{Y}_{k}^{j}\right) \\
& =\left\{G_{i} C x_{k}+\eta_{k}\right\} \oplus\left\{\left(-G_{j} C \hat{X}_{k}^{j}\right) \oplus(-V)\right\} \\
& =G_{j} C\left\{\left\{x_{k}\right\} \oplus\left(-\hat{X}_{k}^{j}\right)\right\} \oplus\left\{\left(G_{i}-G_{j}\right) C x_{k}\right\} \oplus\left\{\eta_{k}\right\} \oplus(-V),
\end{aligned}
$$

where, denoting by $\tilde{X}_{k}^{i j}$ the term $\left\{x_{k}\right\} \oplus\left(-\hat{X}_{k}^{j}\right)$, the residual zonotope can be rewritten as

$$
R_{k}^{i j}=G_{j} C \tilde{X}_{k}^{i j} \oplus\left\{\left(G_{i}-G_{j}\right) C x_{k}\right\} \oplus\left\{\eta_{k}\right\} \oplus(-V) .
$$

Moreover, since $\tilde{X}_{k+1}^{i j}$ is a zonotope, $\tilde{x}_{k+1}^{i j, c}$ and $\tilde{H}_{k+1}^{i j, x}$ are used to denote its center and segment matrix, respectively. Thus, by using (1) and (4), one can have

$$
\begin{aligned}
\tilde{x}_{k+1}^{i j, c} & =x_{k+1}-\hat{x}_{k+1}^{j, c}, \\
\tilde{H}_{k+1}^{i j, x} & =\hat{H}_{k+1}^{j, x},
\end{aligned}
$$


where, using (1), (4) and (7), $\tilde{x}_{k+1}^{i j, c}$ and $\tilde{H}_{k+1}^{i j, x}$ can be further derived as

$$
\begin{aligned}
\tilde{x}_{k+1}^{i j, c} & =\left(A-L_{j} G_{j} C\right) \tilde{x}_{k}^{i j, c}+L_{j}\left(G_{j}-G_{i}\right) C x_{k}-L_{j}\left(\eta_{k}-\eta^{c}\right)+\omega_{k}-\omega^{c}, \\
\tilde{H}_{k+1}^{i j, x} & =\hat{H}_{k+1}^{j, x}=\left[\left(A-L_{j} G_{j} C\right) \hat{H}_{k}^{j, x}-L_{j} H_{\bar{\eta}} H_{\bar{\omega}}\right] .
\end{aligned}
$$

\subsection{Residual-bounding Zonotopes}

In (7), one cannot measure $\tilde{x}_{k}^{i j, c}$ since it involves unmeasurable quantities $\left(\eta_{k}, \omega_{k}\right.$ and $\left.x_{k}\right)$. Thus, to precisely describe the bounds of residual zonotopes, one needs to consider residual-bounding zonotopes which are defined to contain the corresponding residual zonotopes. In order to obtain residual-bounding zonotopes, the following assumption is made for the plant inputs.

Assumption 3.1. The input vector $u_{k}$ of the plant is bounded by a known set $U=\left\{u \in \mathbb{R}^{p}:\left|u-u^{c}\right| \leq\right.$ $\left.\bar{u}, u^{c} \in \mathbb{R}^{p}, \bar{u} \in \mathbb{R}^{p}\right\}$, where the vectors $u^{c}$ and $\bar{u}$ are constant and known.

Notice that, $U$ can be rewritten as a zonotope $U=u^{c} \oplus H_{\bar{u}} \mathbb{B}^{p}$ where $H_{\bar{u}}$ is a $p \times p$ diagonal matrix with the main diagonal composed of $\bar{u}$. For construction of invariant sets, (1a) can be rewritten as

$$
x_{k+1}=A x_{k}+\left[\begin{array}{ll}
B & I
\end{array}\right]\left[\begin{array}{l}
u_{k} \\
\omega_{k}
\end{array}\right] .
$$

Considering $u_{k} \in U$ and $\omega_{k} \in W$, a robust positively invariant (RPI) set, denoted as $X$, can be constructed to confine the states of the dynamics (9) by using Theorem B.1 and Proposition B.1.

Remark 3.1. Any set $X \subset \mathbb{R}^{n}$ invariant with respect to the dynamics (9) is also invariant with respect to all modes (healthy or faulty). This statement holds since the dynamics (9) are not affected by these faults directly or indirectly, as long as the inputs are bounded by the same set.

Since $U$ and $W$ are zonotopes, $X$ is also a zonotope and is denoted as

$$
X=x^{c} \oplus H_{x} \mathbb{B}^{n},
$$

where $x^{c}$ and $H_{x}$ are the center and segment matrix, respectively. By substituting $X, W$ and $V$ into (8) to replace $x_{k}, \omega_{k}$ and $\eta_{k}$, respectively, a bounding zonotope (denoted as $\breve{X}_{k+1}^{i j}$ with the center $\breve{x}_{k+1}^{i j, c}$ and segment matrix $\breve{H}_{k+1}^{i j, x}$ ) to contain $\tilde{X}_{k+1}^{i j}$ can be characterized by its center and segment matrix

$$
\begin{aligned}
\breve{x}_{k+1}^{i j, c} & =\left(A-L_{j} G_{j} C\right) \breve{x}_{k}^{i j, c}+L_{j}\left(G_{j}-G_{i}\right) C x^{c}, \\
\breve{H}_{k+1}^{i j, x} & =\left[\left(A-L_{j} G_{j} C\right) \breve{H}_{k}^{j, x} L_{j}\left(G_{j}-G_{i}\right) C H_{x}-L_{j} H_{\bar{\eta}} H_{\bar{\omega}} L_{j} H_{\bar{\eta}}-H_{\bar{\omega}}\right] .
\end{aligned}
$$

Note that, comparing (8) with (10), it can be observed that (10) is the set-valued version of (8) by considering the bounds of states and uncertainties.

Remark 3.2. As per (8) and (10), if $\tilde{X}_{k^{*}}^{i j} \subseteq \breve{X}_{k^{*}}^{i j}$ holds, $\tilde{X}_{k}^{i j}$ is always contained by $\breve{X}_{k}^{i j}$ for all $k \geq k^{*}$.

Thus, as per (6) and Remark 3.2, one obtains a residual-bounding zonotope $\breve{R}_{k}^{i j}$ to contain $R_{k}^{i j}$, i.e.,

$$
\breve{R}_{k}^{i j}=G_{j} C \breve{X}_{k}^{i j} \oplus\left(G_{i}-G_{j}\right) C X \oplus V \oplus(-V) .
$$


For the center-segment matrix description, an equivalent set description ${ }^{1}$ of $\breve{X}_{k+1}^{i j}$ is obtained as

$$
\breve{X}_{k+1}^{i j}=\left(A-L_{j} G_{j} C\right) \breve{X}_{k}^{i j} \oplus L_{j}\left(G_{j}-G_{i}\right) C X \oplus L_{j} V \oplus(-W) \oplus\left(-L_{j} V\right) \oplus W .
$$

In this paper, the use of residual-bounding zonotopes is twofold. First, they are used for establishing guaranteed FDI conditions. Second, they are used for the transient-state FI after a fault is detected.

\section{Set-based FDI Approaches}

This section gives a brief introduction of the two set-based FDI approaches: interval observer-based and invariant set-based approaches.

\subsection{Interval Observer-based FDI}

According to (Puig et al. 2003; Guerra et al. 2008), the principle of the interval observer-based FD approach is summarized in Proposition 4.1.

Proposition 4.1. During the operation of the $i$-th mode, the interval observer-based FD approach consists in on-line checking whether or not

$$
\mathbf{0} \in R_{k}^{i i}
$$

where $\mathbf{0}$ is the origin and $R_{k}^{i i}$ denotes the residual zonotope estimated by the $i$-th interval observer in the $i$-th mode at time instant $k$.

If the inclusion (13) is violated, it implies that a fault is detected at the current time instant. Otherwise, it is considered that the system still operates in the $i$-th mode.

In (Xu et al. 2013), an FI algorithm using a bank of interval observers has been proposed, where each interval observer is designed to match a considered mode. At each time instant, a collection of residual zonotopes can be obtained corresponding to all interval observers. With respect to Proposition 4.1, the FI algorithm in (Xu et al. 2013) is summarized in Proposition 4.2.

Proposition 4.2. During the operation of the $i$-th mode, if a violation of (13) is detected, a waiting time is timed at the FD time. After the waiting time elapses, FI is implemented by searching an interval observer indexed by $f$ that can estimate residual zonotopes to contain the origin, i.e.,

$$
\mathbf{0} \in R_{k}^{f f}, f \in \mathcal{I} \backslash\{i\} .
$$

Remark 4.1. The definition of the waiting time in Proposition 4.2 can be referred to (Xu et al. 2013).

This paper proposes a new technique to implement sensor FDI by making full use of the systemoperating information from all interval observers (3) and exploiting less conservative FDI conditions.

\subsection{Invariant Set-based FDI}

It is known that a fault-affected system practically switches between several different modes including a healthy one and at least a faulty one. The principle of the invariant set-based FDI technique is to consider invariant sets characterizing each of the different system modes. For each mode, an RPI set characterizing the residual can be constructed (Seron et al. 2012; Olaru et al. 2010; Stoican and Olaru

\footnotetext{
${ }^{1}$ The equivalence of (10) and (12) can be verified by applying zonotope operations into (12) to obtain its center-segment matrix equivalent form, which is the same as (10).
} 
2013). After the system enters into steady state, the residual must be confined by one of these invariant sets, and as long as all invariant sets are disjoint, FDI can be guaranteed.

The FDI technique proposed in this paper includes the invariant set-based FDI principle as a part. Different from the invariant set-based FDI approach, the proposed technique enhances FI performance by complementing its use with the system-operating information from all interval observers. Thus, theoretically, it is possible to loosen FI conditions and improve fault sensitivity and FDI reliability.

\section{Guaranteed FDI Conditions}

This section proposes a novel set-based FDI strategy for sensor faults by combining both interval observer-based and invariant set-based mechanisms.

\subsection{Collecting Process Information}

At each time instant, the system can be monitored in terms of residual zonotopes by means of a bank of interval observers. For brevity, in the $i$-th mode, one defines a vector ${ }^{2}$ of residual zonotopes to collect residual zonotopes estimated by all interval observers at time instant $k$, i.e.,

$$
\mathbf{R}_{k}^{i}=\left(R_{k}^{i 0}, R_{k}^{i 1}, \ldots, R_{k}^{i N}\right)
$$

Table 1. Residual zonotopes

\begin{tabular}{cccccc}
\hline & Interval Observer 0 & $\ldots$ & Interval Observer $i$ & $\ldots$ & Interval Observer $N$ \\
\hline Mode 0 & $R_{k}^{00}$ & $\ldots$ & $R_{k}^{0 i}$ & $\ldots$ & $R_{k}^{0 N}$ \\
$\vdots$ & $\vdots$ & $\ldots$ & $\vdots$ & $\ldots$ & $\vdots$ \\
Mode $i$ & $R_{k}^{i 0}$ & $\ldots$ & $R_{k}^{i i}$ & $\ldots$ & $R_{k}^{i N}$ \\
$\vdots$ & $\vdots$ & $\ldots$ & $\vdots$ & $\ldots$ & $\vdots$ \\
Mode $N$ & $R_{k}^{N 0}$ & $\ldots$ & $R_{k}^{N i}$ & $\ldots$ & $R_{k}^{N N}$ \\
\hline
\end{tabular}

Table 2. Limit sets of residual-bounding zonotopes

\begin{tabular}{cccccc}
\hline & Interval Observer 0 & $\ldots$ & Interval Observer $i$ & $\ldots$ & Interval Observer $N$ \\
\hline Mode 0 & $\breve{R}_{\infty}^{00}$ & $\ldots$ & $\breve{R}_{\infty}^{0 i}$ & $\ldots$ & $\breve{R}_{\infty}^{0 N}$ \\
$\vdots$ & $\vdots$ & $\ldots$ & $\vdots$ & $\ldots$ & $\vdots$ \\
Mode $i$ & $\breve{R}_{\infty}^{i 0}$ & $\ldots$ & $\breve{R}_{\infty}^{i i}$ & $\ldots$ & $\breve{R}_{\infty}^{i N}$ \\
$\vdots$ & $\vdots$ & $\ldots$ & $\vdots$ & $\ldots$ & $\vdots$ \\
Mode $N$ & $\breve{R}_{\infty}^{N 0}$ & $\ldots$ & $\breve{R}_{\infty}^{N i}$ & $\ldots$ & $\breve{R}_{\infty}^{N N}$ \\
\hline
\end{tabular}

Remark 5.1. In this paper, the indices of rows and columns of tables and matrices start from 0 . The index 0 corresponds to the healthy mode and interval observer.

Furthermore, if considering residual zonotopes corresponding to all modes and interval observers, one can collect all available real-time system-operating information, which is presented in Table 1. Except the real-time process-operating information conveyed by residual zonotopes estimated by a bank of interval observers, there exists additional off-line process information provided by the limit sets of all residual-bounding zonotopes. Table 2 collects the limit sets of all residual-bounding zonotopes, i.e., the

\footnotetext{
${ }^{2}$ Because, in any mode, residual zonotopes are obtainable, without ambiguity, the notation $\mathbf{R}_{k}^{i}$ corresponding to the $i$-th mode can be generally replaced by the notation $\mathbf{R}_{k}$.
} 
smallest sets of residual zonotopes. Each row of Table 2 corresponds to a mode. Thus, from Table 2, a matrix describing all modes can be extracted as

$$
\mathbf{M}=\left[\begin{array}{ccccc}
\breve{R}_{\infty}^{00} & \ldots & \breve{R}_{\infty}^{0 i} & \ldots & \breve{R}_{\infty}^{0 N} \\
\vdots & \vdots & \vdots & \vdots & \vdots \\
\breve{R}_{\infty}^{i 0} & \ldots & \breve{R}_{\infty}^{i i} & \ldots & \breve{R}_{\infty}^{i N} \\
\vdots & \vdots & \vdots & \vdots & \vdots \\
\breve{R}_{\infty}^{N 0} & \ldots & \breve{R}_{\infty}^{N i} & \ldots & \breve{R}_{\infty}^{N N}
\end{array}\right]
$$

Remark 5.2. Comparing Table 1 with Table 2 , it is known that, during the steady-state operation, each entry in Table 2 is the set of the corresponding entry in Table 1 such as $R_{k}^{i 0} \subseteq \breve{R}_{\infty}^{i 0}$ when $k$ is sufficiently large. Similarly, furthermore, one should have $\mathbf{R}_{k}^{i} \subseteq \mathbf{M}^{i},(i \in \mathcal{I})$, where $\mathbf{M}^{i}$ is the $i$-th row of $\mathbf{M}$ and $\subseteq$ should be understood elementwise.

Table 3. Transformation of Table 2

\begin{tabular}{cccccc}
\hline & Interval Observer 0 & $\ldots$ & Interval Observer $i$ & $\ldots$ & Interval Observer $N$ \\
\hline Mode 0 & 1 & $\ldots$ & $1 \backslash 0$ & $\ldots$ & $1 \backslash 0$ \\
$\vdots$ & $\vdots$ & $\ldots$ & $\vdots$ & $\ldots$ & $\vdots$ \\
Mode $i$ & $1 \backslash 0$ & $\ldots$ & 1 & $\ldots$ & $1 \backslash 0$ \\
$\vdots$ & $\vdots$ & $\ldots$ & $\vdots$ & $\ldots$ & $\vdots$ \\
Mode $N$ & $1 \backslash 0$ & $\ldots$ & $1 \backslash 0$ & $\ldots$ & 1 \\
\hline
\end{tabular}

In order to simplify Table 2, one defines the following rules. First, if $\mathbf{0} \in \breve{R}_{\infty}^{i j}$, the position of $\breve{R}_{\infty}^{i j}$ is labelled as 1 . Second, if $\mathbf{0} \notin \breve{R}_{\infty}^{i j}$, the position of $\breve{R}_{\infty}^{i j}$ is labelled as 0 . After applying these rules for Table 2, Table 3 is generated, containing binary information in concordance with the above logical propositions. Notice that, as per the interval observer-based FDI principle in Proposition 4.1, $0 \in R_{k}^{i i} \subseteq$ $\breve{R}_{\infty}^{i i}$ (for all $i \in \mathcal{I}$ ) should always hold during the steady-state operation of the $i$-th mode. Similarly, from Table 3, a matrix describing all collected off-line mode information can be obtained as

$$
\mathbf{I}=\left[\begin{array}{ccccc}
1 & \ldots & 1 \backslash 0 & \ldots & 1 \backslash 0 \\
\vdots & \vdots & \vdots & \vdots & \vdots \\
1 \backslash 0 & \ldots & 1 & \ldots & 1 \backslash 0 \\
\vdots & \vdots & \vdots & \vdots & \vdots \\
1 \backslash 0 & \ldots & 1 \backslash 0 & \ldots & 1
\end{array}\right]
$$

\subsection{FDI Conditions}

After collecting all available information as in (17), one should analyze how much information is useful and how much is available but redundant/unnecessary for FDI. It is mentioned that all diagonal entries of (17) are 1 because $\mathbf{0} \in R_{k}^{i i}$ and $R_{k}^{i i} \subseteq \breve{R}_{k}^{i i}$ always holds during the steady-state operation of the $i$-th mode as in Proposition 4.1. For the non-diagonal entries of (17), one does not know theoretically whether or not they can contain $\mathbf{0}$ in advance. In this case, one should consider two possibilities.

- For the non-diagonal entries with 0 , one has $\mathbf{0} \notin R_{\infty}^{i j}(i \neq j)$, because $R_{\infty}^{i j} \subseteq \breve{R}_{\infty}^{i j}$ and $\mathbf{0} \notin \breve{R}_{\infty}^{i j}$ imply $\mathbf{0} \notin R_{\infty}^{i j}$.

- For the non-diagonal entries with 1 , one cannot have $\mathbf{0} \in R_{\infty}^{i j}(i \neq j)$, because $R_{\infty}^{i j} \subseteq \breve{R}_{\infty}^{i j}$ and $\mathbf{0} \in \breve{R}_{\infty}^{i j}$ do not guarantee $\mathbf{0} \in R_{\infty}^{i i}$.

Since the non-diagonal entries with 1 in the matrix $\mathbf{I}$ cannot guarantee that their corresponding residual zonotopes in Table 1 contain $\mathbf{0}$, these entries are not useful for the proposed FDI approach and the 
residual-bounding zonotopes corresponding to these entries should be discarded in order to reduce computational complexity. Comparatively, the non-diagonal entries with 0 can guarantee that the residual zonotopes corresponding to them do not contain $\mathbf{0}$, which are useful for the proposed FDI approach.

In this paper, the proposed FDI approach is based on the combined use of residual zonotopes and residual-bounding zonotopes. Residual zonotopes estimated by interval observers can always be obtained in real time. Residual-bounding zonotopes are generated especially for the transient-state FI after a fault is detected. The limit sets of residual-bounding zonotopes (in Table 2) are mainly used for establishing guaranteed FDI conditions, which are used for off-line pre-checking whether or not the considered faults are detectable and isolable in advance. For the sake of explaining how to establish these FDI conditions, one takes the following matrix $\mathbf{I}$ as an example, i.e.,

$$
\mathbf{I}=\left[\begin{array}{llll}
1 & 0 & 0 & 0 \\
1 & 1 & 0 & 1 \\
1 & 1 & 1 & 1 \\
1 & 0 & 0 & 1
\end{array}\right]
$$

The example (18) considers four modes corresponding to the four rows of the matrix, respectively. Moreover, four interval observers should be designed to monitor these modes. According to the aforementioned analysis, the system information of the example (18) useful for FDI can be described by

$$
\mathbf{I}^{\prime}=\left[\begin{array}{cccc}
1 & 0 & 0 & 0 \\
\times & 1 & 0 & \times \\
\times & \times & 1 & \times \\
\times & 0 & 0 & 1
\end{array}\right]
$$

In this example, if one wants to guarantee that all the four modes are detectable and isolable after their occurrences, it should be assured that any two rows of the matrix (19) are distinguishable. Notice that when verifying the distinguishability of the four modes, one does not consider the entries with $\times$ of the matrix (19). Instead, only the entries with 0 or 1 are considered. Moreover, one cares about the columns (only columns without containing entries with $\times$ ) of any two rows, as long as there exists one column whose two entries are different (i.e., one is 0 and the other one is 1 ), it is guaranteed that the two modes corresponding to these two rows are detectable and isolable.

Similarly, taking (19) as an example, when considering Row 0 and 1 of the matrix (19), one can see that Column 1 and 2 of the two rows should be considered to see whether or not the two rows are distinguishable. It can be observed that, in Column 1 of the two rows, the two entries corresponding to Row 0 and 1 are 0 and 1, respectively, which are different. This means that, only by using these two entries of Column 1, Row 0 and 1 (i.e., Mode 0 and 1) can be distinguished. Based on the same principle, for any other rows, one can make the similar analysis to know whether or not they are distinguishable.

Remark 5.3. For simplicity, one takes the example (19) to show the principle of the FDI conditions of this proposed FDI approach. Thus, for real applications, one should obtain the corresponding matrices $\mathbf{I}$ and $\mathbf{I}^{\prime}$ of these applications and use the method shown in this example to analyze whether or not all considered modes (healthy or faulty) are detectable and isolable.

Based on this principle shown by the example (19), the FDI conditions for the proposed FDI approach can be established in Proposition 5.1.

Proposition 5.1. For all modes (healthy or faulty), a matrix $\mathbf{I}$ as in (18) corresponding to residualbounding zonotopes can be obtained. Furthermore, based on the matrix $\mathbf{I}$, a simplified matrix $\mathbf{I}^{\prime}$ as in (19) can be obtained. If for any two rows in the matrix $\mathbf{I}^{\prime}$, there exists one column without $\times$ entries, whose two column entries are different from each other, the two modes corresponding to these two rows are detectable and isolable after their occurrences.

Proof. For any two rows of the matrix $\mathbf{I}^{\prime}$, as long as there exist one column of these two rows whose two 
entries are different from each other, it means that the two modes corresponding to these two rows are distinguishable from each other. When the two entries of this column are different from each other (one is 0 and the other one is 1 ), it means that as the time elapses, the residual zonotopes estimated by the interval observer corresponding to this column will either always contain the origin or not, which will only accord with one entry of this column (i.e., only one row out of the two rows). Thus, the switching between these two modes is always detectable and isolable. Furthermore, if any two rows satisfy these conditions, all modes are detectable and then isolable.

Notice that, if there exists an FDI algorithm that can identify these differences of rows in the matrix $\mathbf{I}^{\prime}$, it implies that Proposition 5.1 can be used as guaranteed FDI conditions of this FDI algorithm .

\section{FDI Algorithm}

This section proposes an algorithm to implement sensor FDI under the FDI conditions proposed in Proposition 5.1.

\subsection{Fault Detection}

To explain the proposed FD principle, it is assumed that the system is in the $i$-th mode. Thus, a vector $\mathbf{R}_{k}$ (i.e., $\mathbf{R}_{k}^{i}$ in the $i$-th mode) composed of residual zonotopes estimated by all interval observers can be obtained in real time. If the system operates at steady state, residual zonotopes should be bounded by the limit sets of residual-bounding zonotopes corresponding to the $i$-th mode, i.e.,

$$
\mathbf{R}_{k} \subseteq \mathbf{M}^{i}
$$

Thus, whenever (20) is violated elementwise, it implies that a fault ${ }^{3}$ has occurred. Additionally, after the system enters into the operation of the $i$-th mode, residual zonotopes can be used to test whether or not

$$
\mathbf{0} \in \mathbf{R}_{k}
$$

where (21) should be understood elementwise. After testing (21) at each time instant, one can obtain an $(N+1)$-dimensional fault signature vector $\mathcal{F}_{i}(i$ denotes that, before FD, the system is in the $i$-th mode) full of 0 and 1 , where, as in (17), 0 represents that residual zonotopes estimated by the interval observer corresponding to the vector entry with 0 do not contain the origin while 1 has the opposite explanation. Thus, for FD, one should compare this real-time vector with the $i$-th row of the matrix $\mathbf{I}^{\prime}$ (the elements with $\times$ of this row should be omitted). If this real-time vector matches the $i$-th row of the matrix $\mathbf{I}^{\prime}$, it is considered that the system is still in the $i$-th mode. Otherwise, it means that a fault has occurred.

Remark 6.1. A criteria simpler than (21) can also be used to detect faults in the $i$-th mode, i.e., testing whether or not

$$
\mathbf{0} \in R_{k}^{i i}
$$

holds. However, under the FDI conditions in Proposition 5.1, (22) is only sufficient but not necessary for FD. Thus, if (22) is violated, it means that the system becomes faulty. Otherwise, one cannot assure whether or not a mode switching has occurred. This implies that (22) may only be able to detect a part of the considered modes. Instead, under the FDI conditions in Proposition 5.1, (21) can detect all the considered modes. Thus, at each time instant, for simplicity, one can first test the $i$-th component of (21)

\footnotetext{
${ }^{3}$ Notice that, for the proposed FDI approach, if they can also be used for detecting recovery process, then one still uses the terms such as fault detection and isolation and fault occurrence to describe the general mode switching.
} 
(i.e., (22)) to make a quick FD decision. If (22) is violated, it implies a mode switching. Otherwise, then test the rest of components of (21) to confirm whether or not a mode switching has occurred.

Proposition 6.1. If the system is at steady-state regime of its $i$-th mode, FD can be performed by simultaneously testing (20) and (21). As long as either of them detects anomaly, it implies that a fault has occurred. Otherwise, it is considered that the system is still in the $i$-th mode.

Proof. At steady state of the $i$-th mode, once residual zonotopes enter into their sets, they remain inside. Only when the system mode is changed by faults, a violation of (20) becomes possible. Regarding (21), the FD principle is also similar by testing the consistency as explained. Thus, whenever (20) or (21) is violated, it implies that a fault has occurred.

It is known that testing both (20) or (21) allows to implement FD. But based on the FDI conditions proposed in Proposition 5.1, it is not known whether the FD strategy (20) can guarantee that all the considered modes are detectable or not. But, the FD strategy (21) can guarantee that. If one chooses to test both of them on-line for FD, this combination may be more sensitive to some faults. But, the combination has high computational complexity. Thus, an alternative is that only the FD strategy (21) is used in the proposed FD approach, which has less computational complexity. For applications, the designers can make a selection between these two ways according to the particular requirements.

\subsection{Fault Isolation}

In this paper, the proposed FI algorithm is based on residual zonotopes and residual-bounding zonotopes, which is also composed of two different FI strategies (transient-state and steady-state). In this subsection, these two FI strategies are presented, respectively.

In order to explain the FI algorithm, it is assumed that the system is at steady state of the $i$-th mode and a fault is detected at time $k_{d}$, which implies that the system switches to a new mode different from the $i$-th one after the FD time instant $k_{d}$. At the FD time $k_{d}$, residual zonotopes $R_{k_{d}}^{f j}\left(f \in \mathcal{I}_{i}=\mathcal{I} \backslash\{i\}\right)$ can always be obtained, where $f$ denotes the index of a new and unknown mode.

As per Proposition B.1 in Appendix B, using a starting set to initialize (12), a set sequence can be generated by iterating the dynamics. As $k$ tends to infinity, the set sequence finally converges to a fixed set (i.e., the mRPI set $\breve{X}_{\infty}^{i j}$ of $\tilde{X}_{k}^{i j}$ indicated in (8) for the $j$-th interval observer in the $i$-th mode).

Thus, at the FD time $k_{d}$, by initializing all the dynamics (12) corresponding to each interval observer $\left(j \in \mathcal{I}_{i}\right)$ with a starting set, a group of set sequences can be generated and a group of the corresponding residual-bounding zonotopic set sequences can be simultaneously obtained by using (11). In this paper, a starting set for the $j$-th interval observer in the $f$-th mode is denoted as $\bar{X}_{k_{d}}^{f j}$, which should satisfy

$$
\bar{X}_{k_{d}}^{f j} \supseteq \tilde{X}_{k_{d}}^{f j}
$$

Thus, a collection of starting sets should be constructed to initialize the corresponding set-based dynamics (12), each of which corresponds to one interval observer.

Remark 6.2. Although the residual-bounding zonotope sequences corresponding to all interval observers under all modes can be generated by using their corresponding starting sets at the FD time $k_{d}$, this FI approach only uses the $N$ residual-bounding zonotope sequences $\breve{R}_{k}^{j j}\left(k \geq k_{d}\right.$ and $\left.j \in \mathcal{I}_{i}\right)$ for less computational complexity.

By means of the dynamics (12), with the corresponding starting sets at the FD time $k_{d}$, the set sequences $\breve{X}_{k}^{j j}\left(k \geq k_{d}\right.$ and $\left.j \in \mathcal{I}_{i}\right)$ can be generated, which will always contain the state estimation error sets $\tilde{X}_{k}^{j j}\left(k \geq k_{d}\right)$ according to Remark 3.2 if the current mode is also the $j$-th one. Furthermore, by using the expression (11), the corresponding residual-bounding zonotope sequences $\breve{R}_{k}^{j j}$ can be computed, respectively. Similarly, the generated residual-bounding zonotope sequence $\breve{R}_{k}^{j j}$ will always contain the residual zonotopes estimated by the $j$-th interval observer if the current system is also in the $j$-th mode. 
However, if the current system is not in the $j$-th mode, the sequence $\breve{R}_{k}^{j j}\left(k \geq k_{d}\right)$ may not contain the residual zonotopes $R_{k}^{f j}$ even at the FD time $k_{d}$. This can be explained by the fact that residual-bounding zonotopes depend on the system modes (see (11)). At time instant $k_{d}$, even though the starting sets of (12) for all interval observers $j \in \mathcal{I}_{i}$ respectively satisfy

$$
\bar{X}_{k_{d}}^{f j} \supseteq \tilde{X}_{k_{d}}^{f j}, f \neq i
$$

it cannot still guarantee $\breve{R}_{k_{d}}^{j j} \supseteq R_{k_{d}}^{f j}$, where $\breve{R}_{k_{d}}^{f j}$ is computed as

$$
\breve{R}_{k_{d}}^{f j}=G_{j} C \bar{X}_{k_{d}}^{j j} \oplus V \oplus(-V) .
$$

Thus, whenever a fault is detected, the proposed transient FI strategy generates $N$ residual-bounding zonotope sequences $\breve{R}_{k}^{j j}$ ( $k \geq k_{d}$ and $j \in \mathcal{I}_{i}$ ), each of which corresponds to one candidate mode. This implies that, among the $N$ residual-bounding zonotope sequences, there exists at least one (i.e., the one matching the current after-fault mode) that can always contain the residual zonotopes estimated by its corresponding interval observer for all $k \geq k_{d}$. Based on this fact, the transient-state FI strategy is summarized in Proposition 6.2.

Proposition 6.2. In the $i$-th mode, when a fault is detected at the FD time $k_{d}$, $N$ residual-bounding zonotope sequences described by the candidate-mode set $\mathcal{I}_{i}$ can be generated by initializing (12) with their corresponding starting sets and using (11), the transient-state FI strategy consists in searching a mode by testing whether or not

$$
R_{k}^{f j} \subseteq \breve{R}_{k}^{j j}, j \in \mathcal{I}_{i}, \text { for all } k \geq k_{d},
$$

holds in real time. If a violation of (25) corresponding to the $j$-th interval observer is detected, one removes the index $j$ from the candidate-mode set $\mathcal{I}_{i}$. Testing and removing are repeated until the time instant when the set $\mathcal{I}_{i}$ remains only one element or the time window used to describe the transition completely elapses. This time instant and this unique element indicates the FI time and fault, respectively.

Remark 6.3. Since sensor faults can immediately affect the system outputs, a sensor fault is possible to be directly isolated by this FI strategy with initialization at the FD time $k_{d}$ (see (23) and (24)).

Remark 6.4. It is possible that, even though the proposed transient-state FI algorithm is persistently executed, there always exist at least two elements in $\mathcal{I}_{i}$ during the corresponding time window. This implies that an accurate FI decision may not be obtained by this transient-state FI strategy during the transition. In this paper, in order to describe the transition induced by faults and the persistent time of the use of this transient-state FI strategy, a proper time window should be defined in advance.

In order to avoid the situation indicated in Remark 6.4, one also proposes a steady-state FI strategy to complement the transient-state FI strategy proposed in Proposition 6.2 by using residual zonotopes and testing the inclusions between residual zonotopes and $\mathbf{0}$ after the time window. By testing these inclusions, one can obtain the fault signature vector $\mathcal{F}_{i}$. Finally, by matching $\mathcal{F}_{i}$ with the rows of $\mathbf{I}^{\prime}$, if one row of $\mathbf{I}^{\prime}$ can match $\mathcal{F}_{i}$, then the index of this row indicates the fault. This steady-state FI strategy is summarized in Proposition 6.3.

Proposition 6.3. After applying the transient-state FI strategy over a defined time window, if there still exist at least two elements in $\mathcal{I}_{i}$, FI can still be guaranteed by searching an unique row in $\mathbf{I}^{\prime}$ that can match the fault signature vector $\mathcal{F}_{i}$ and the FI decision is indicated by the index of this row.

Proof. Under Proposition 5.1, as $k$ tends to infinity, all residual-bounding zonotope sequences converge to their corresponding fixed sets (i.e., the elements of the matrix $\mathbf{M}$ ). Since if residual-bounding zonotope sequences do not contain $\mathbf{0}$, it is guaranteed that its corresponding residual zonotopes in Table 1 at steady state do not contain $\mathbf{0}$ too. Moreover, in the case that an interval observer matches the current system 
mode, the residual zonotopes estimated by this interval observer can contain $\mathbf{0}$ at steady state. This implies that, under Proposition 5.1, the fault signature vector is different in different modes and can match one and only one row of the matrix $\mathbf{I}^{\prime}$. Thus, FI can be guaranteed by comparing $\mathcal{F}_{i}$ with the rows of the matrix $\mathbf{I}^{\prime}$ on-line.

To explain how to obtain $\mathcal{F}_{i}$, one still uses the example given by (18) and (19). Firstly, one assumes that the system is in the healthy mode at the beginning, after a fault is detected, one knows that all the faults 1, 2 and 3 are candidates. With the matrix (19), it can be observed that the faults 1 and 2 can be distinguished by using residual zonotopes estimated by the observer 2 , the faults 1 and 3 by the observer 1 and the faults 2 and 3 also by the observer 2 . This means that, after a fault is detected, one only needs to use residual zonotopes estimated by the interval observers 1 and 2, while the other two interval observers 0 and 3 are not necessary. From the computational complexity point of view, $\mathcal{F}_{0}$ should be a two-dimensional vector whose elements are composed of the binary information by testing the inclusions between residual zonotopes estimated by the interval observers 1 and 2 and the origin. But, for simplicity, one can also define $\mathcal{F}_{0}$ as a four-dimensional vector, whose four elements are obtained by testing the inclusions between residual zonotopes estimated by the four interval observers and the origin. But, finally, only the 1-st and 2-nd elements of $\mathcal{F}_{0}$ are used to compare with the 1-st and 2-nd elements of Row 1, 2 and 3 of the matrix (19) for FI, while the 0-th and 3-rd elements of $\mathcal{F}_{0}$ are not useful.

Remark 6.5. The whole FI algorithm simultaneously includes these two FI strategies in Propositions 6.2 and 6.3. The former may be able to isolate faults during the transition induced by faults but without FI guarantees, while the latter with FI guarantees requires longer FI time after the transition.

\subsection{Construction of Starting Sets}

In Section 6.2, it can be observed that the starting sets for residual-bounding zonotope sequences are crucial for the transient-state FI strategy. Here, an idea to construct these starting sets is given. Firstly, by using a zonotope $V_{0}=H_{\bar{\eta}} \mathbb{B}^{q}$, in the unknown mode $f$, for the $j$-th interval observer, the corresponding residual zonotope indicated in (5) can be transformed into

$$
R_{k_{d}}^{f j}=G_{j} C \tilde{X}_{k_{d}}^{f j} \oplus\left\{\left(G_{f}-G_{j}\right) C x_{k_{d}}\right\} \oplus\left\{\eta_{k_{d}}-\eta^{c}\right\} \oplus\left(-V_{0}\right), f \neq i, j \neq i,
$$

By adding $-\left(\eta_{k_{d}}-\eta^{c}\right)$ and $-\left(G_{f}-G_{j}\right) C x_{k_{d}}$ to both sides, the previous equation turns into

$$
R_{k_{d}}^{f j} \oplus\left\{-\left(\eta_{k_{d}}-\eta^{c}\right)\right\} \oplus\left\{\left(G_{j}-G_{f}\right) C x_{k_{d}}\right\}=G_{j} C \tilde{X}_{k_{d}}^{f j} \oplus\left(-V_{0}\right) .
$$

Considering $\eta_{k_{d}}-\eta^{c} \in V_{0}$ and $x_{k_{d}} \in X$, one further has

$$
G_{j} C \tilde{X}_{k_{d}}^{f j} \oplus\left(-V_{0}\right) \subseteq R_{k_{d}}^{f j} \oplus\left(-V_{0}\right) \oplus\left\{\left(G_{j}-G_{f}\right) C X\right\} .
$$

Since $V_{0}$ can be removed from both sides of the previous equation, one can obtain

$$
G_{j} C \tilde{X}_{k_{d}}^{f j} \subseteq R_{k_{d}}^{f j} \oplus\left\{\left(G_{j}-G_{f}\right) C X\right\} .
$$

It can be observed that the right side of (29) is dependent of modes. But, before fault isolation, it is not possible to know the new mode. Thus, one should construct initial sets without being affected by modes. Here, one should consider three cases.

- If $j \neq f$ and $j=0$, one always has

$$
G_{j}-G_{f}=\operatorname{diag}\left(0, \ldots, 0,1-g_{f}, 0, \ldots, 0\right) .
$$

where $g_{f}$ is the $f$-th diagonal element of $G_{f}$ that models the $f$-th sensor fault. 
- If $j \neq f$ and $j \neq 0$, one always has

$$
G_{j}-G_{f}=\operatorname{diag}\left(0, \ldots, 0, g_{j}-1,0, \ldots, 0,1-g_{f}, 0, \ldots, 0\right),
$$

where $g_{j}$ is the $j$-th diagonal element of $G_{j}$ that models the $j$-th sensor fault.

- If $j=f$, one always has

$$
G_{j}-G_{f}=0
$$

By summarizing the aforementioned three cases, one can obtain that, if $j=0$, one always has

$$
G_{j}-G_{f} \in G_{f j}=\operatorname{diag}\left(\left[0,1-g_{1}\right],\left[0,1-g_{2}\right], \ldots,\left[0,1-g_{q}\right]\right),
$$

while if $j \neq 0$, one always has

$$
G_{j}-G_{f} \in G_{f j}=\operatorname{diag}\left(\left[0,1-g_{1}\right], \ldots,\left[0,1-g_{j-1}\right], 1-g_{j},\left[0,1-g_{j+1}\right], \ldots,\left[0,1-g_{q}\right]\right) .
$$

where $G_{f j}$ is a diagonal interval matrix that can always include $G_{j}-G_{f}$ inside its interval as long as the mode switching from the $j$-th mode occurs. Thus, one can further have

$$
\left(G_{j}-G_{f}\right) C X \subseteq G_{f j} C X
$$

where, considering that $X$ is a zonotope and $G_{f j}$ is an interval matrix, with Properties A.6 and A.7, $G_{f j} C X$ can be over-approximated by a zonotope denoted as

$$
Z_{f j}=z^{f j, c} \oplus H_{z}^{f j} \mathbb{B}_{z}^{s^{f j}}
$$

where $s^{f j}$ is the order of $Z_{f j}$. Thus, in order to remove the effect of modes, a solution is to further transform (29) into

$$
G_{j} C \tilde{X}_{k_{d}}^{f j} \subseteq R_{k_{d}}^{f j} \oplus Z_{f j}
$$

Since $R_{k_{d}}^{f j}$ is zonotope, the term $R_{k_{d}}^{f j} \oplus Z_{f j}$ can be rewritten into the zonotopic form

$$
R_{k_{d}}^{f j} \oplus Z_{f j}=r_{k_{d}}^{f j, c} \oplus H_{k_{d}}^{f j, r} \mathbb{B}^{f j, r}
$$

where $s_{k_{d}}^{f j, r}$ is the corresponding zonotope order, and $r_{k_{d}}^{f j, c}$ and $H_{k_{d}}^{f j, r}$ can be respectively derived as

$$
\begin{aligned}
r_{k_{d}}^{f j, c} & =y_{k_{d}}-\hat{y}_{k_{d}}^{c, y}+z^{f j, c}, \\
H_{k_{d}}^{f j, r} & =\left[\begin{array}{ll}
\hat{H}_{k_{d}}^{j, y} & H_{z}^{f j}
\end{array}\right] .
\end{aligned}
$$

With the help of the zonotopic form of $R_{k_{d}}^{f j} \oplus Z_{f j}$, (31) is rewritten as

$$
G_{j} C \tilde{X}_{k_{d}}^{f j} \oplus\left\{-r_{k_{d}}^{f j, c}\right\} \subseteq H_{k_{d}}^{f j, r} \mathbb{B}_{k_{d}}^{s^{f j, r}} .
$$

Using $F(l), H_{k_{d}}^{f j, r}(l)$ and $r_{k_{d}}^{f j, c}(l)$ to denote the $l$-th rows of $G_{j} C$ and $H_{k_{d}}^{f j, r}$ and the $l$-th component of $r_{k_{d}}^{f j, c}$, respectively, one can obtain a group of inequalities corresponding to (34), where the $l$-th inequality 
can be written as

$$
\left|F(l) \tilde{x}_{k}^{f j}-r_{k_{d}}^{f j, c}(l)\right| \leq\left\|H_{k_{d}}^{f j, r}(l)\right\|_{1}, l=1,2, \ldots, q,
$$

where $\tilde{x}_{k}^{f j}$ represents the elements that satisfy (35).

Remark 6.6. It can be observed that the description (35) is more conservative than (34), which means, if the $q$ inequalities of (35) can determine a closed set, the closed set can fully contain $\tilde{X}_{k_{d}}^{f j}$ in (34).

Assumption 6.1. For the $j$-th interval observer, $\tilde{X}^{j}$ denotes a given zonotope determined by the physical constraints of the system and can always bound $\tilde{X}_{k}^{i j}$ for all $k \geq 0$.

As per Property A.4 in Appendix A, each inequality out of the $q$ inequalities of (35) generally determines a strip and the $q$ inequalities together can form a closed set that contains $\tilde{X}_{k_{d}}^{f j}$. However, there exist two possible cases that depend on the system dynamics.

- If the $q$ inequalities themselves can form a closed set, then the formed set can contain $\tilde{X}_{k_{d}}^{f j}$.

- If the $q$ inequalities cannot form a closed set (i.e., there are not enough strips such that their intersection can lead to a closed set), then $\tilde{X}^{j}$ indicated in Assumption 6.1 can be further used to construct a closed set that can contain $\tilde{X}_{k_{d}}^{f j}$.

However, because Property A.4 can only compute a zonotope approximation of the intersection of a zonotope and a strip, for the $j$-th interval observer, $\tilde{X}^{j}$ has to be used as the initial zonotope of Property A.4 for both cases. As seen in Section 6.2, for the $j$-th interval observer, the constructed starting zonotope for the residual-bounding zonotope sequence is denoted as $\bar{X}_{k_{d}}^{f j}$.

Remark 6.7. In a particular case, if the matrix $G_{j} C$ is invertible, (31) can be directly transformed into

$$
\tilde{X}_{k_{d}}^{f j} \subseteq\left(G_{j} C\right)^{-1}\left(R_{k_{d}}^{f j} \oplus Z_{f j}\right)
$$

where $\left(G_{j} C\right)^{-1}$ denotes the inverse of $G_{j} C$ and $\left(G_{j} C\right)^{-1}\left(R_{k_{d}}^{f j} \oplus Z_{f j}\right)$ is directly used as $\bar{X}_{k_{d}}^{f j}$.

At the FD time $k_{d}$, the proposed FI approach constructs a group of starting sets for generating $N$ corresponding residual-bounding set sequences $\breve{R}_{k}^{j j}\left(j \in \mathcal{I}_{i}\right)$. As derived before, for the $j$-th interval observer, its residual-bounding set sequence is generated by initializing its corresponding dynamics (12) with the starting set

$$
\breve{X}_{k_{d}}^{j j}=\bar{X}_{k_{d}}^{f j}, j \in \mathcal{I}_{i}
$$

Remark 6.8. In order to construct the starting sets at the FD time $k_{d}$, this subsection proposed a method by using Property A.4 or Property A.4 with $\tilde{X}^{j}$ in Assumption 6.1 . However, one can still introduce another method based on Property A.5 to construct the starting sets, where Property A.5 computes a zonotopic approximation of the intersection of a polytope and a zonotope. Using Property A.5, one can use all $q$ strips (35) as a whole (i.e., a polytope) and compute the zonotopic approximation of the intersection of this polytope and a zonotope to construct the starting sets.

\subsection{Practical FDI Algorithm}

According to the aforementioned discussions, based on the FDI conditions in Proposition 5.1, faults can be detected with Proposition 6.1. Whenever a fault is detected, after applying the proposed FI strategies in Propositions 6.2 and 6.3, theoretically, the worst case is that the fault can only be isolated at infinity because the FDI conditions are built by means of the limit sets of residual-bounding zonotopes. However, from the practical point of view, it is impossible to obtain residual-bounding zonotopes at infinity. Thus, in order to establish the FDI conditions and implement the FDI approach, one has to consider the 
approximations of the limit sets of residual-bounding zonotopes.

According to Theorem B.1 in Appendix B, for the set-based dynamics (12), the mRPI set $\breve{X}_{\infty}^{i j}$ can be approximated by an RPI set denoted as $\dot{X}^{i j}$ with an arbitrarily approximate precision to $\breve{X}_{\infty}^{i j}$. Thus, the set $\breve{R}_{\infty}^{i j}$ can be approximated by the corresponding set

$$
\stackrel{\circ}{R}^{i j}=G_{j} C \stackrel{\circ}{X}^{i j} \oplus\left(G_{i}-G_{j}\right) C X \oplus V \oplus(-V) .
$$

Notice that whenever $\tilde{X}_{k}^{i j}$ goes into and stays inside $\dot{X}^{i j}, R_{k}^{i j}$ also goes into and stays inside $\stackrel{\circ}{R}^{i j}$. Since all sets (i.e., $\stackrel{R}{R}^{i j}$ ) can be computed off-line, all entries of Table 2 is over-approximated by Table 4.

Table 4. Sets of residual zonotopes

\begin{tabular}{|c|c|c|c|c|c|}
\hline & Interval Observer 0 & $\ldots$ & Interval Observer $i$ & $\ldots$ & Interval Observer $N$ \\
\hline Mode 0 & $\stackrel{\circ}{R}^{00}$ & $\ldots$ & $\stackrel{\circ}{R}^{0 i}$ & $\cdots$ & $\stackrel{\circ}{R}^{0 N}$ \\
\hline$\vdots$ & $\vdots$ & $\ldots$ & $\vdots$ & $\ldots$ & : \\
\hline Mode $i$ & $\stackrel{\circ}{R}^{i 0}$ & $\ldots$ & $\stackrel{\circ}{R}^{i i}$ & $\ldots$ & $\stackrel{\circ}{R}^{i N}$ \\
\hline Mode $N$ & $\stackrel{\circ}{R}^{N 0}$ & $\begin{array}{l}\cdots \\
\cdots\end{array}$ & $\stackrel{\circ}{R}^{N i}$ & $\begin{array}{l}\cdots \\
\cdots\end{array}$ & $\stackrel{\circ}{R}^{N N}$ \\
\hline
\end{tabular}

According to the definition of the mRPI set in Definition B.2 in Appendix B, $\breve{X}_{\infty}^{i j} \subseteq \dot{X}^{i j}$ and $\breve{R}_{\infty}^{i j} \subseteq \stackrel{\circ}{R}^{i j}$ hold. This implies that after initializing (12), the set sequence of the corresponding residualbounding zonotopes will finally converge to its corresponding set (i.e., $\stackrel{\circ}{R}^{i j}$ ) as $k$ increases.

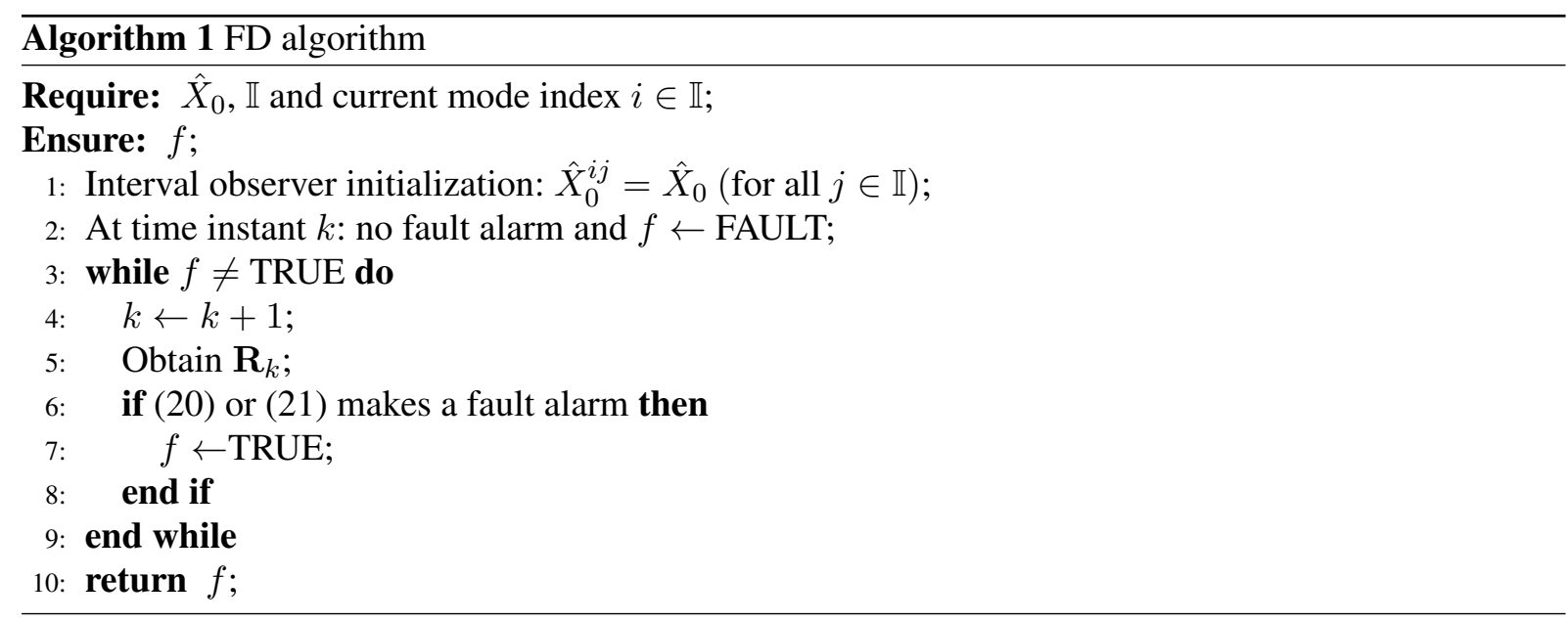

Thus, as long as the given approximate precision is sufficiently high, Table 4 can be used to replace Table 2 for verifying the FDI conditions in Proposition 5.1. Moreover, Table 3 can be derived from Table 4. Furthermore, based on Table 4, for the $i$-th mode, one defines a vector

$$
\stackrel{\circ}{\mathbf{R}}^{i}=\left(\stackrel{\circ}{R}^{i 0}, \stackrel{\circ}{R^{i 1}}, \ldots, \stackrel{\circ}{R}^{i N}\right),
$$

which is used for Algorithm 1 to carry out the proposed FD strategy. The proposed FI algorithm is a combination of the two FI strategies in Propositions 6.2 and 6.3. Practically, once a fault is detected, this FI algorithm firstly starts up the FI strategy in Proposition 6.2. If the FI strategy in Proposition 6.2 cannot isolate the fault within a defined time window, the FI algorithm will terminate it and then the FI strategy in Proposition 6.3 is then started to guarantee FI at steady state. Thus, in the proposed FI algorithm, the defined time window for the transient-state FI strategy is used as a switching mechanism between the two FI strategies in Propositions 6.2 and 6.3.

According to (12), after the initialization required to obtain residual-bounding zonotope sequences on-line, the differences between the set values of residual-bounding zonotopes at different time instants 
are dependent of the term $\left(A-L_{j} G_{j} C\right) \breve{X}_{k}^{i j}$, i.e., the eigenvalues of $A-L_{j} G_{j} C$ and the starting set $\breve{X}_{k_{d}}^{i j}$ of initialization. Because all eigenvalues of $A-L_{j} G_{j} C$ are inside the unit circle (i.e., the dynamics of residual-bounding zonotopes (12) are stable), after the transition induced by a fault, all residual-bounding zonotope sequences finally enter into steady state and can sufficiently and asymptotically approximate their corresponding limit sets (i.e., $\breve{R}_{\infty}^{i j}$ ). This implies that, at steady state, the difference between the set values of a residual-bounding zonotope sequence at different time instants will gradually decrease. This fact allows to define a proper time window as mentioned in Remark 6.4 as the switching mechanism between the two FI strategies.

Definition 6.1. The time window $T$ starting from the FD time instant $k_{d}$ is defined at least as the maximal settling time of the dynamics of all interval observers such that the proposed FI strategy in Proposition 6.3 can guarantee reliable FI.

Remark 6.9. The transitions between different modes are determined by the eigenvalues of the corresponding dynamics. Theoretically, it can also be assessed by the settling time of interval observers. Most importantly, a proper time window can always be selected by a sufficient number of simulations.

As per Definition 6.1 and Remark 6.9, a mechanism of switching between the proposed FI strategies in Propositions 6.2 and 6.3 is introduced by the time window $T$ defined in Definition 6.1 and the proposed FI algorithm is summarized as follows:

(1) Once a fault is detected by Proposition 6.1, the transient-state FI strategy in Proposition 6.2 is firstly started to isolate the fault within the time window $T$.

(2) After $T$, if the fault is still not isolated, then the first FI strategy is terminated and the FI algorithm starts up the second FI strategy proposed in Proposition 6.3 for FI at steady state .

(3) After the second FI strategy enters in operation, at each time instant, the inclusions between residual zonotopes and $\mathbf{0}$ are tested, and the testing results are used to compare with the off-line inclusion information contained in each row of $\mathbf{I}^{\prime}$.

(4) If at a time instant, the obtained results (i.e., $\mathcal{F}_{i}$ ) match one row of $\mathbf{I}^{\prime}$, the index of this row indicates the fault (this index is assumed as $f$ ).

(5) In order to improve reliability of the FI decision given in Step $4, \mathbf{R}_{k} \subseteq \stackrel{\circ}{\mathbf{R}}^{f}$ is also tested. If $\mathbf{R}_{k} \subseteq \stackrel{\circ}{\mathbf{R}}^{f}$ holds, the FI decision of Step 4 can be confirmed. Otherwise the second FI strategy in Proposition 6.3 is repeated again.

Remark 6.10. Both Steps 4 and 5, respectively considering the invariant set-based and the interval observer-based FI principles, are used to make FI decisions. However, the core step is Step 4, while Step 5 is an assistant step. For simplicity, one can omit Step 5 and only use Step 4.

Notice that, the proposed FDI approach is based on the combination of invariant sets and interval observers. By this combination, the conservatism of the FDI conditions should be reduced in some sense because of the use of all available system-operating information from all the corresponding interval observers. Comparing with the purely interval observer-based or the purely invariant set-based approach, it can be observed that the FDI effectiveness and reliability is possible to be improved.

\section{Illustrative Example}

In this paper, the second pitch system of a wind turbine benchmark proposed in (Odgaard et al. 2009) is used as the case study. The continuous-time dynamics of this subsystem can be clearly found in (Stoican et al. 2011), i.e.,

$$
\begin{aligned}
\dot{x}(t) & =A x(t)+B u(t), \\
y(t) & =C x(t) .
\end{aligned}
$$


In this subsystem, two sensors are used to measure the pitch position $y(t)$, whose measurements are

$$
\begin{aligned}
& y_{1}(t)=G_{1}\left(C x(t)+\eta_{1}(t)\right), \\
& y_{2}(t)=G_{2}\left(C x(t)+\eta_{2}(t)\right),
\end{aligned}
$$

where $y_{1}(t)$ and $y_{2}(t)$ denote the measurements of the first and second sensors, respectively, $\eta_{1}(t)$ and $\eta_{2}(t)$ are the corresponding measurement noises, $G_{1}$ and $G_{2}$ models the fault in the first and second sensors, respectively. If $G_{1}$ (or $G_{2}$ ) is the identity matrix, it means that the corresponding sensor is healthy. Otherwise, it implies that the corresponding sensor is faulty. Besides, the control action of the subsystem is designed as

$$
u(t)=u^{r e f}(t)+u^{f}(t)
$$

where $u^{r e f}(t)$ is the given reference input and $u^{f}(t)$ is the feedback signal ${ }^{4}$ with a form $u^{f}(t)=y(t)-$ $0.5\left(y_{1}(t)+y_{2}(t)\right)$. Furthermore, one can equivalently reformulate (40) and (41) of the pitch system into a compact form, which entirely describes the pitch system, i.e.,

$$
\begin{aligned}
\dot{x}(t) & =A x(t)+B u(t), \\
y_{12}(t) & =G_{12} C x(t)+\eta(t),
\end{aligned}
$$

where

$$
y_{12}(t)=\left[\begin{array}{l}
y_{1}(t) \\
y_{2}(t)
\end{array}\right], G_{12}=\left[\begin{array}{l}
G_{1} \\
G_{2}
\end{array}\right], \eta(t)=\left[\begin{array}{cc}
G_{1} & 0 \\
0 & G_{2}
\end{array}\right]\left[\begin{array}{l}
\eta_{1}(t) \\
\eta_{2}(t)
\end{array}\right]
$$

The sets of the noises of the sensors are described by $\overline{\eta_{1}}=0.8, \eta_{1}{ }^{c}=0, \overline{\eta_{2}}=0.8$ and $\eta_{2}{ }^{c}=0$, which follows the form as in (2). The parameters of the second pitch system are given as

$$
A=\left[\begin{array}{cc}
-13.33 & -123.43 \\
1 & 0
\end{array}\right], B=\left[\begin{array}{l}
1 \\
0
\end{array}\right], C=\left[\begin{array}{ll}
0 & 123.43
\end{array}\right] .
$$

Remark 7.1. Since the noises are Gaussian, the aforementioned bounds are empirical values according to the fact that, usual choices for Gaussian distribution, are the band $[-3 \sigma, 3 \sigma]$ with probability of $99 \%$, or band $[-6 \sigma, 6 \sigma]$ with probability of $99.99 \%$.

The sampling time of the pitch system is $0.01 \mathrm{~s}$. After discretization, the system parameters are

$$
A_{d}=\left[\begin{array}{cc}
0.867 & -1.234 \\
0.01 & 1
\end{array}\right], B_{d}=\left[\begin{array}{c}
0.01 \\
0
\end{array}\right], C_{d}=\left[\begin{array}{ll}
0 & 123.43
\end{array}\right] .
$$

In this case study, one considers two faults in the two sensors, respectively, and it is assumed that one and only one sensor becomes faulty at any given time (note that one can also consider that two sensors become faulty simultaneously). Thus, the system should have three different sensor modes: healthy, fault in the first sensor and fault in the second sensor, which are respectively modelled as

$$
G_{12}^{0}=\left[\begin{array}{l}
1 \\
1
\end{array}\right], G_{12}^{1}=\left[\begin{array}{c}
0.1 \\
1
\end{array}\right], G_{12}^{2}=\left[\begin{array}{c}
1 \\
0.1
\end{array}\right] .
$$

\footnotetext{
${ }^{4}$ The form of this feedback signal completely respects the original structure of the second pitch system in the wind turbine benchmark proposed in (Odgaard et al. 2009).
} 

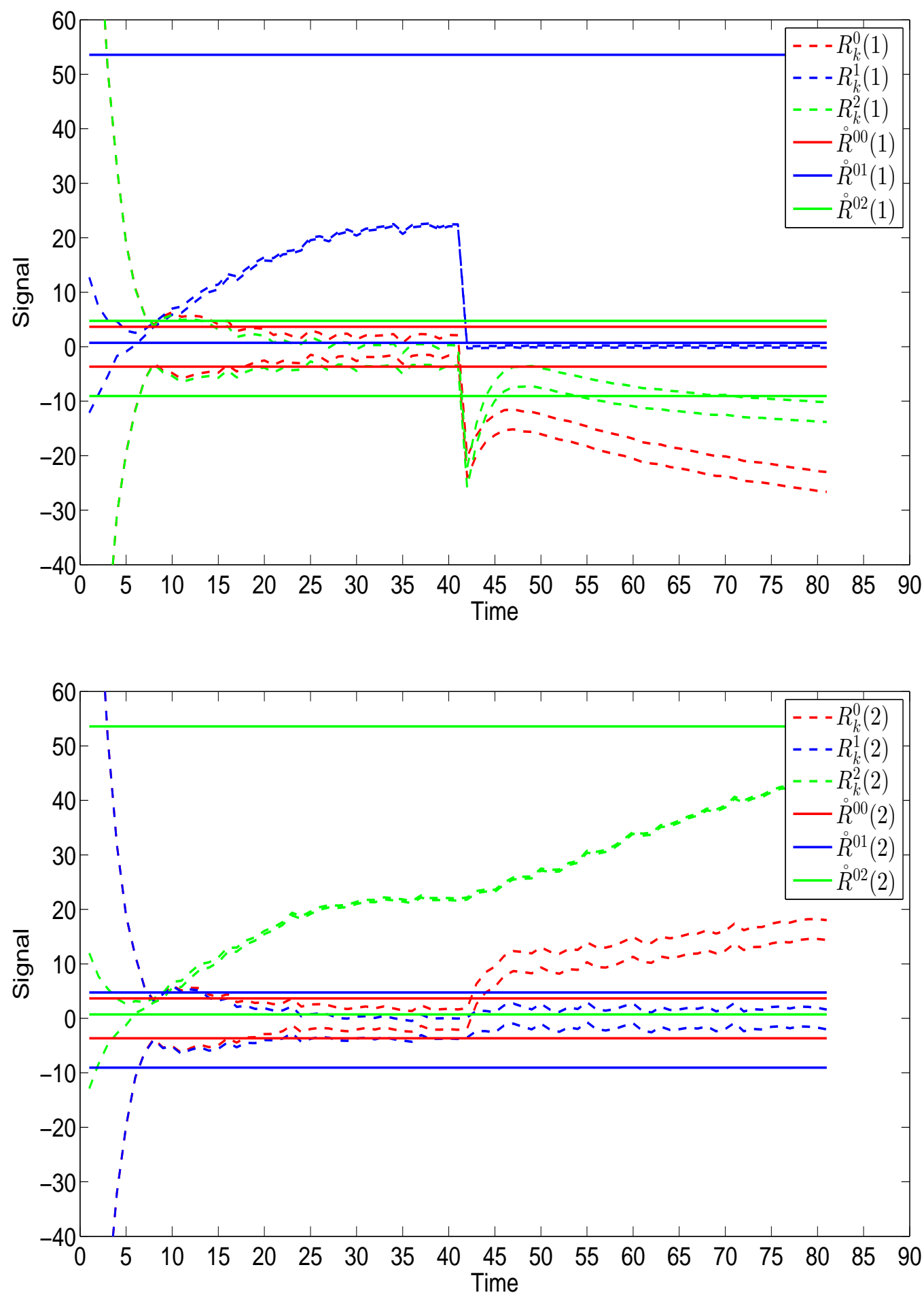

Figure 2. FD of the fault 1

In this paper, one assumes that the reference input ${ }^{5}$ of the pitch system varies in the operating range $u^{r e f}(t) \in\left[10^{\circ}, 30^{\circ}\right]$. Furthermore, as per (42), by simulating the pitch system with a time span of $10^{6} \mathrm{~s}$, an empirical bound of $u(t)$ is obtained as $u(t) \in[8.561,52.2314]$. Thus, as per the discretized model, Theorem B.1 and Proposition B.1 in Appendix B, an RPI approximation of the mRPI set of states is

\footnotetext{
${ }^{5}$ In this case study, as an example, the reference input $u^{r e f}(t)$ is chosen as a sinusoidal signal.
} 
constructed by iterating 150 times from an initial state RPI set, which is denoted as

$$
X=\left[\begin{array}{c}
0 \\
0.2463
\end{array}\right] \oplus\left[\begin{array}{cc}
2.3934 & 0 \\
0 & 0.2252
\end{array}\right] \mathbb{B}^{2}
$$
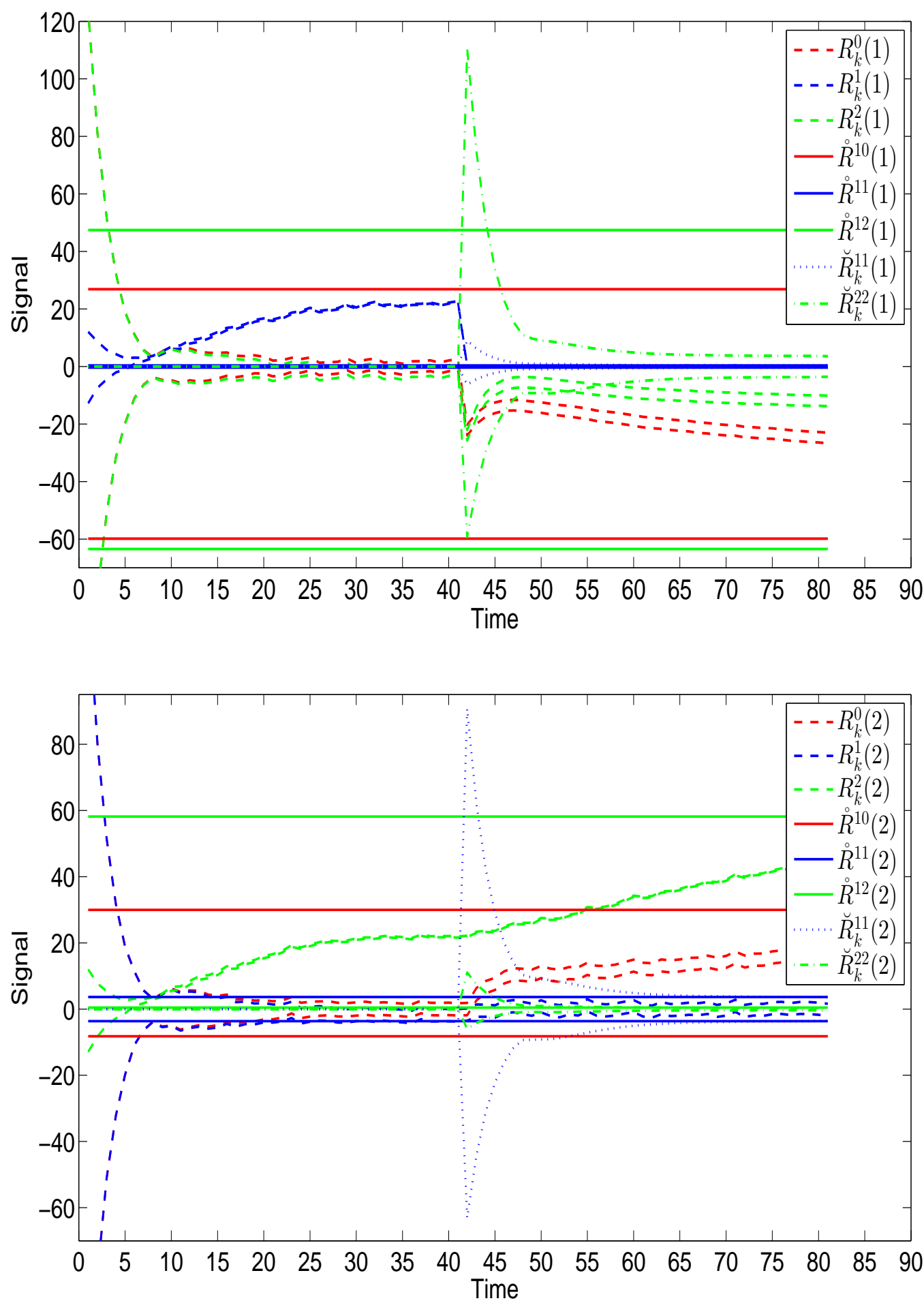

Figure 3. FI of the fault 1 
Based on the discrete-time model from (43), three interval observers indicated as in (3), a healthy interval observer and two faulty interval observers, are respectively designed to monitor the modes. The initial state of the system and the initial zonotope of interval observers are respectively given as

$$
x_{0}=\left[\begin{array}{l}
0 \\
0
\end{array}\right], \hat{X}_{0}=\left[\begin{array}{l}
0 \\
0
\end{array}\right] \oplus\left[\begin{array}{ll}
1 & 0 \\
0 & 1
\end{array}\right] \mathbb{B}^{2} .
$$

In order to obtain FDI guarantees, one has to check the corresponding FDI conditions in Proposition 5.1. As per Theorem B.1 and Proposition B.1 in Appendix B, for each mode, the approximations of the limit sets (i.e., $\breve{R}_{\infty}^{i j}$ ) of the relevant residual-bounding zonotopes, indicated in Table 4 , are obtained by iterating the dynamics (12) 120 times with initial RPI sets of $\tilde{X}_{k}^{i j}$. These approximations are presented as follows:

- For the healthy mode:

$$
\begin{aligned}
& \stackrel{\circ}{R}^{00}=([-3.6507,3.6507],[-3.6507,3.6507])^{T}, \\
& \stackrel{\circ}{R}^{01}=([0.7196,53.5725],[-9.0443,4.7407])^{T}, \\
& \stackrel{\circ}{R}^{02}=([-9.0443,4.7407],[0.7196,53.5725])^{T} .
\end{aligned}
$$

- For the fault in the first sensor:

$$
\begin{aligned}
& \stackrel{\circ}{R}^{10}=([-59.8823,26.8929],[-8.2239,29.9569])^{T}, \\
& \stackrel{\circ}{R}^{11}=([-0.3651,0.3651],[-3.6507,3.6507])^{T}, \\
& \stackrel{\circ}{R}^{12}=([-63.4498,47.4594],[0.4448,58.1507])^{T} .
\end{aligned}
$$

- For the fault in the second sensor:

$$
\begin{aligned}
& \stackrel{\circ}{R}^{20}=([-8.2239,29.9569],[-59.8823,26.8929])^{T}, \\
& \stackrel{\circ}{R}^{21}=([0.4448,58.1507],[-63.4498,47.4594])^{T}, \\
& \stackrel{\circ}{R}^{22}=([-3.6507,3.6507],[-0.3651,0.3651])^{T} .
\end{aligned}
$$

By analyzing these approximations, one can obtain the matrix shown in Table 5, which collects the off-line system-operating information corresponding to all different modes and interval observers.

Table 5. Available off-line system information

\begin{tabular}{lccc}
\hline & Interval Observer 0 & Interval Observer 1 & Interval Observer 2 \\
\hline Mode 0 & 1 & 0 & 0 \\
Mode 1 & 1 & 1 & 0 \\
Mode 2 & 1 & 0 & 1 \\
\hline
\end{tabular}

Furthermore, based on Table 5, one can obtain the matrix $\mathbf{I}^{\prime}$, which is shown as

$$
\mathcal{I}^{\prime}=\left[\begin{array}{lll}
1 & 0 & 0 \\
\times & \mathbf{1} & 0 \\
\times & \mathbf{0} & 1
\end{array}\right]
$$

It can be checked that the matrix $\mathbf{I}^{\prime}$ in (44) satisfies the FDI conditions proposed in Proposition 5.1. As observed in (44), all the non-diagonal entries with 1 are omitted. After omitting these entries, it is observed that the three rows of the matrix $\mathbf{I}^{\prime}$ are different from each other, which means that three 

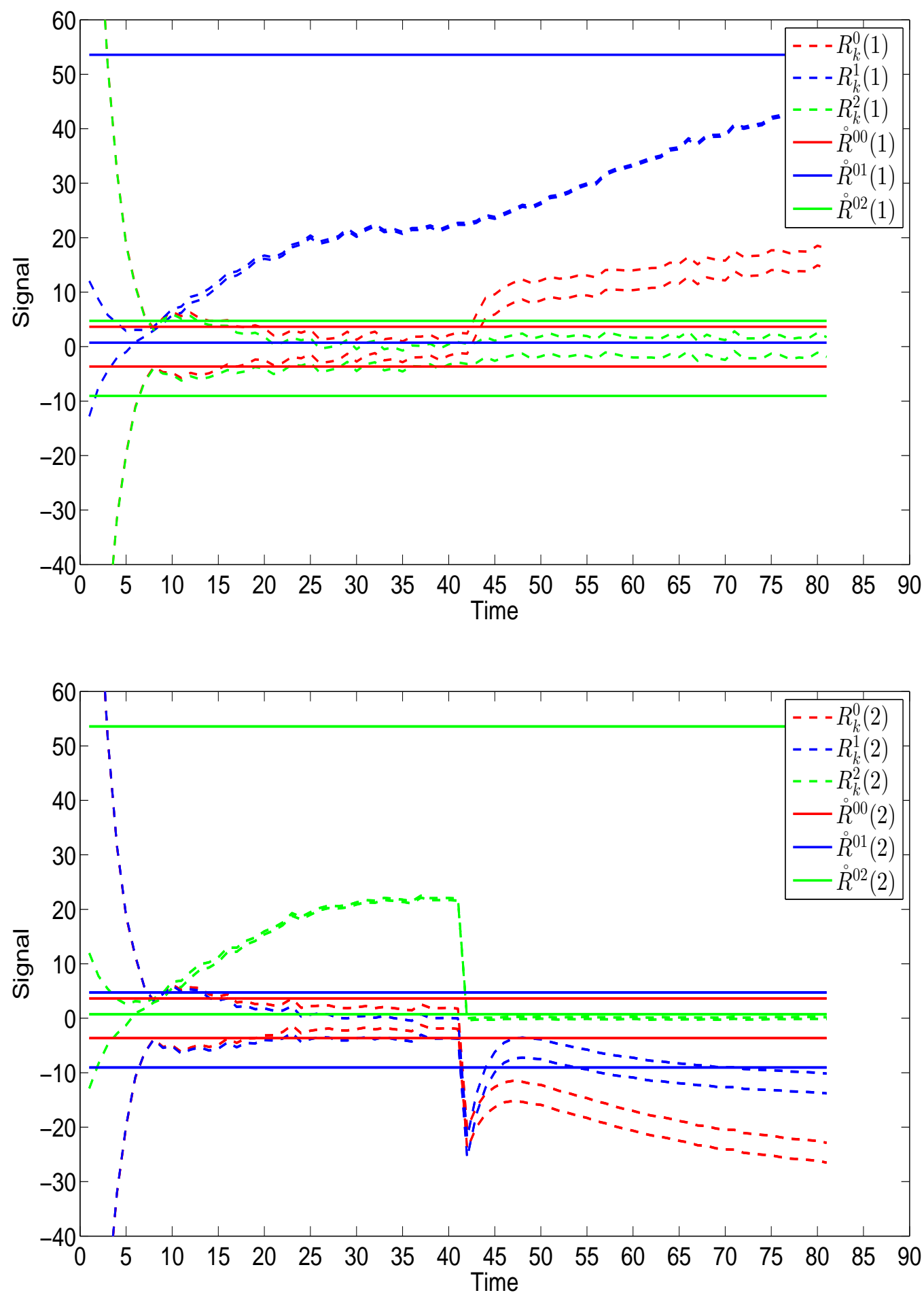

Figure 4. FD of the fault 2

modes are distinguishable from each other. Thus, whenever, if a considered mode has occurred, it can be guaranteed that the occurrence of the mode can be detected and then isolated by the FDI approach.

With loss of generality, this example only considers the dynamical process of the system from healthy to faulty. Thus, it is not necessary to take Row 0 corresponding to the healthy mode into account in terms of FI of the two sensor faults. According to the FD strategy in Proposition 6.1, at most, $R_{k}^{0}, R_{k}^{1}, R_{k}^{2}$, $\stackrel{\circ}{R}^{00}, \stackrel{\circ}{R}^{01}$ and $\stackrel{\circ}{R}^{02}$ are used for FD implementation. Moreover, according to the proposed FI strategies in 
Propositions 6.2 and 6.3, one only needs to obtain $R_{k}^{1}$ or $R_{k}^{2}$ to distinguish the two sensor-fault modes and to test the inclusions between the origin and them, respectively. Eventually, FI can be done by comparing the tested inclusion results with the rows of $\mathbf{I}^{\prime}$ and a matched row of $\mathbf{I}^{\prime}$ will indicate the fault.

According to the proposed FI approach in Proposition 6.2, by initializing (12), one can obtain the corresponding residual-bounding zonotope sequences $\breve{R}_{k}^{11}$ and $\breve{R}_{k}^{22}$ for isolating the fault 1 and 2 during the transient-state operation. But one should notice that the transient-state FI strategy in Proposition 6.2 cannot be guaranteed by the FDI conditions in Proposition 5.1. This implies that the transient-state FI strategy may be able to isolate faults during the transition or not, which depends on the faults themselves. Besides, for the two considered scenarios, the sets for the transient-state FI strategy in Assumption 6.1 are empirically given by simulations as

$$
\tilde{X}^{0}=\left[\begin{array}{l}
0 \\
0
\end{array}\right] \oplus\left[\begin{array}{ll}
3 & 0 \\
0 & 1
\end{array}\right] \mathbb{B}^{2}
$$

The time window in Definition 6.1 is given as five steps, and in order to compute the starting zonotopes for the initialization for residual-bounding zonotope sequences, $\lambda$ in Property A.4 in Appendix A is given as $\lambda=\left[\begin{array}{ll}0.005 & 0.005\end{array}\right]^{T}$.

Remark 7.2. The selection of $\lambda$ is important for the proposed approach, which affects the volume of the obtained starting zonotopes by Property A.4. Please refer to (Alamo et al. 2005) for details.

In this example, one considers the same scenario for both sensor faults: from time instants 1 to 40 , the system is healthy, then a fault occurs at time instant 41 and the system is in the faulty operation from the instants 41 to 80 . Thus, the simulations for the two sensor faults are done, respectively, and the diagnostic results of the faults in the first and second sensors are shown in Figures 2, 3, 4 and 5.

Remark 7.3. In the figures, $R_{k}^{i}(1)$ and $R_{k}^{i}(2)$ denote the first and second components of the residual zonotope $R_{k}^{i}$ estimated by the $i$-th interval observer at time instant $k$. Similarly, $\stackrel{\circ}{R}^{i j}(1)$ and $\stackrel{R}{R}^{i j}(2)$ denote the first and second components of the approximation $R^{i j}$ of $R_{\infty}^{i j}$ corresponding to the $j$-th interval observer in the $i$-th mode.

According to the FD principle in Proposition 6.1 and the simulation results shown in Figure 2, it can be observed that a fault is detected at time instant 42 (i.e, $\mathbf{0} \notin R_{42}^{0}, R_{42}^{0} \nsubseteq \stackrel{\circ}{R^{00}}, R_{42}^{1} \nsubseteq \stackrel{\circ}{R}^{01}$ and $R_{42}^{2} \nsubseteq \stackrel{\circ}{R}^{02}$ ). Furthermore, according to the proposed transient-state FI approach in Proposition 6.2 and the simulation results shown in Figure 3, the first fault is isolated at time instant 42 because of $R_{42}^{1} \subseteq \breve{R}_{42}^{11}$ and $R_{42}^{2} \nsubseteq \breve{R}_{42}^{22}$. This indicates that the first fault is isolated at the same time when it is detected, which means no time delay between FD and FI (see Remark 6.3). Additionally, in order to show the steadystate FI strategy proposed in Proposition 6.3, one should wait a defined five-step time window. Thus, one should test whether or not $\mathbf{0} \in R_{47}^{1}$ (or/and $\mathbf{0} \in R_{47}^{2}$ ) holds. As shown in Figure 3, it is seen that $\mathbf{0} \in R_{47}^{1}$ (or/and $\mathbf{0} \notin R_{47}^{2}$ ) holds, which matches Row 1 of the matrix $\mathbf{I}^{\prime}$. This implies that the fault is in the first sensor, which provides the same FI decision with the transient-state FI strategy.

Remark 7.4. The size of $\bar{X}_{k_{d}}^{f j}$ affects the quickness of the transient-state FI strategy in Proposition 6.2, but the effect is not decisive. Because, even though the transient-state FI strategy cannot isolate faults within the time window, the proposed FI algorithm can still use the steady-state FI strategy in Proposition 6.3 to guarantee to isolate faults after the time window.

Similarly, FDI of the second fault is illustrated in Figures 4 and 5. In Figure 4, it is seen that a fault is detected at time instant 42 (i.e., $\mathbf{0} \notin R_{42}^{0}, R_{42}^{0} \not \subset \stackrel{R}{R}^{00}, R_{42}^{1} \not \subset \stackrel{\circ}{R}^{01}$ and $R_{42}^{2} \nsubseteq \stackrel{R^{02}}{0}$ ). In Figure 5, it can be observed that $R_{42}^{1} \nsubseteq \subseteq \breve{R}_{42}^{11}$ and $R_{42}^{2} \subseteq \breve{R}_{42}^{22}$ hold, which means that the second sensor fault has occurred by the transient-state FI strategy. Moreover, if considering the steady-state FI strategy in Proposition 6.3, one can observe that $\mathbf{0} \notin R_{47}^{11}$ (or/and $\mathbf{0} \in R_{47}^{21}$ ) holds, which is in accordance with Row 2 of the matrix $\mathbf{I}^{\prime}$ in (44), which means that at time instant 47 , the fault can also be isolated by the steady-state FI strategy. 

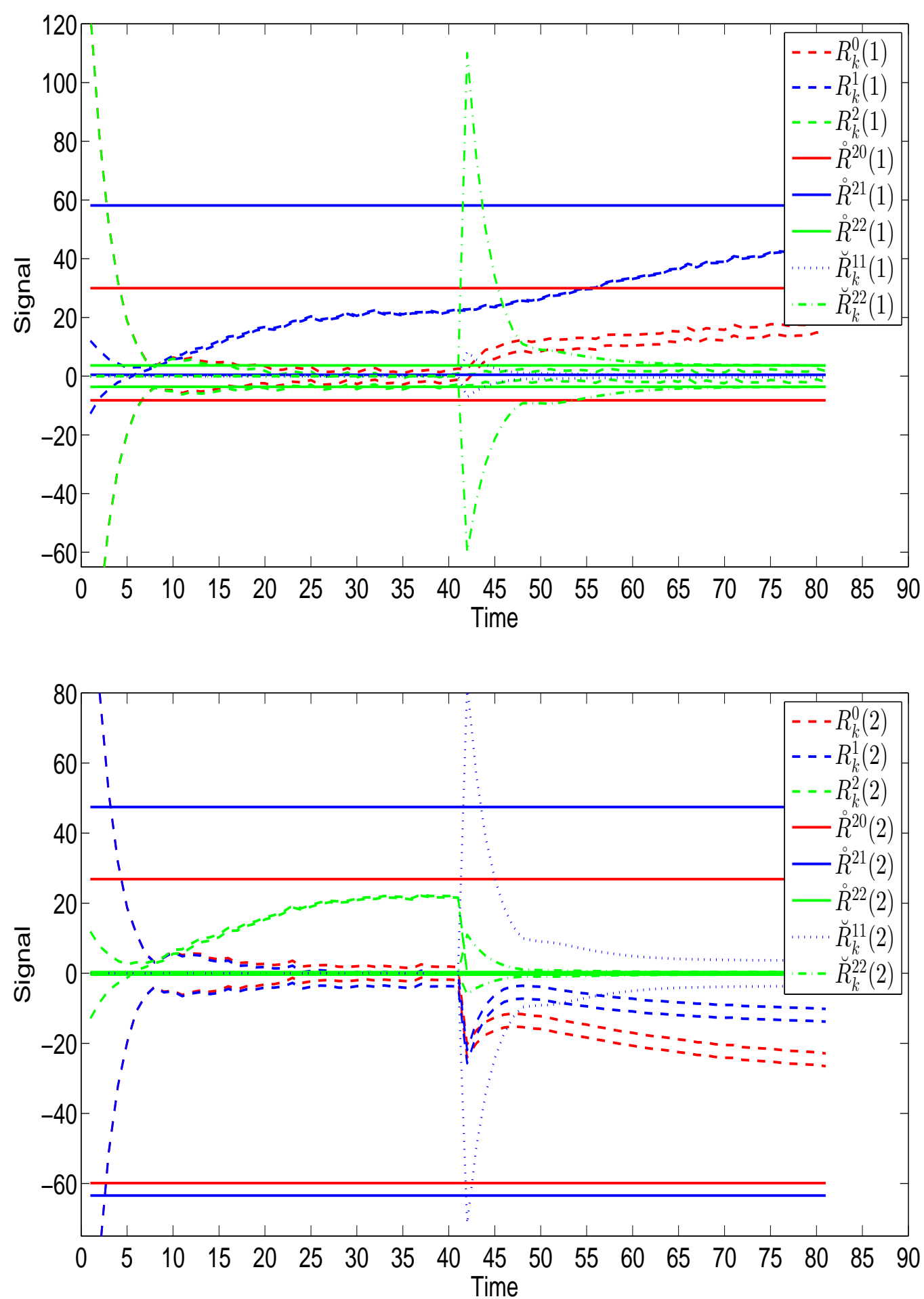

Figure 5. FI of the fault 2

Remark 7.5. According to the proposed FI algorithm, after a fault is detected, the fault should be either in the first or second sensor. Thus, as seen in the matrix $\mathbf{I}^{\prime}$ in (44), one only needs to use the residual zonotopes estimated by either the interval observer 1 or 2 (or both of these two interval observers), which corresponds to the bold and italic columns of Row 1 and 2 of the matrix $\mathbf{I}^{\prime}$, respectively. This can reduce computational complexity of FI. 


\section{Conclusion}

This paper proposes a sensor FDI approach based on set-theoretic approaches. In this approach, two different set-theoretic FDI mechanisms are simultaneously used, i.e., invariant set-based and interval observer-based mechanisms. This approach implements the combination of invariant sets and interval observers for sensor FDI, and both FD and FI decisions are based on the results of the two FDI mechanisms. A bank of interval observers are used to monitor the system operation in real time and collect system-operating information for FDI, and invariant sets describing the steady-state behaviors of the system are used to establish guaranteed FDI conditions. Furthermore, in order to reduce the computational complexity, the available but redundant system-operating information is discarded by a new strategy. By means of these strategies, the objective is to use as much available information from all interval observers as possible to improve fault sensitivity but simultaneously discard all redundant information to simplify the FDI algorithm. Besides, another objective of the proposed approach is to isolate faults as soon as possible. For sensor faults, this approach can isolate faults during the transition induced by faults and the fastest FI case is that sensor faults are isolated at the same time instant when they are detected. Due to more system-operating information used, this approach should have less conservative guaranteed FDI conditions comparing with the existing invariant set-based or interval observer-based approaches. Additionally, for simplicity, although this paper only considers the linear time-invariant systems and the given magnitudes of sensor faults, it could be extended to the system with parametric uncertainties and unknown but bounded faults in principle.

\section{Appendix A. Zonotopes}

According to (Alamo et al. 2005; Le et al. 2013; Kühn 1998), one summarizes the relevant results of zonotopes used in this paper here.

Definition A.1. An $r$-order zonotope $Z$ is defined as $Z=g \oplus H \mathbb{B}^{r}$, where $g$ and $H$ are the center and segment matrix (also generator matrix), respectively.

Definition A.2. The interval hull $\square Z$ of a zonotope $Z=g \oplus H \mathbb{B}^{r} \subset \mathbb{R}^{n}$ is the smallest box containing $X$ and the interval hull is denoted as $\square Z=\left\{x|| x_{i}-g_{i} \mid \leq\left\|H_{i}\right\|_{1}\right\}$, where $H_{i}$ is the $i$-th row of $H$, and $x_{i}$ and $g_{i}$ are the $i$-th components of $x$ and $g$, respectively.

Property A.1. Given two zonotopes $Z_{1}=g_{1} \oplus H_{1} \mathbb{B}^{r_{1}} \subset \mathbb{R}^{n}$ and $Z_{2}=g_{2} \oplus H_{2} \mathbb{B}^{r_{2}} \subset \mathbb{R}^{n}$, the Minkowski sum of them is $Z_{1} \oplus Z_{2}=\left\{g_{1}+g_{2}\right\} \oplus\left[\begin{array}{ll}H_{1} & H_{2}\end{array}\right] \mathbb{B}^{r_{1}+r_{2}}$.

Property A.2. Given a zonotope $Z=g \oplus H \mathbb{B}^{r} \subset \mathbb{R}^{n}$ and a compatible matrix $K, K Z=K g \oplus K H \mathbb{B}^{r}$.

Property A.3. Given a zonotope $Z=g \oplus H \mathbb{B}^{r} \subset \mathbb{R}^{n}$ and an integer $s$ (with $n<s<r$ ), denote by $\hat{H}$ the matrix resulting from the reordering of the columns of $H$ in decreasing Euclidean norm. $Z \subseteq g \oplus\left[\begin{array}{ll}\hat{H}_{T} & Q\end{array}\right] \mathbb{B}^{s}$ where $\hat{H}_{T}$ is obtained from the first $s-n$ columns of $\hat{H}$ and $Q \in \mathbb{R}^{n \times n}$ is a diagonal matrix whose elements satisfy $Q_{i i}=\sum_{j=s-n+1}^{r}\left|\hat{H}_{i j}\right|, i=1, \ldots, n$.

Property A.4. Given a zonotope $Z=g \oplus H \mathbb{B}^{r} \subset \mathbb{R}^{n}$, a strip $S=\left\{x \in \mathbb{R}^{n}|| c x-d \mid \leq \sigma\right\}$ and a vector $\lambda \in \mathbb{R}^{n}$, then $Z \cap S \subseteq \hat{Z}(\lambda)=\hat{g}(\lambda) \oplus \hat{H}(\lambda) \mathbb{B}^{r+1}$ where $\hat{g}(\lambda)=g+\lambda(d-c g)$ and $\hat{H}(\lambda)=[(I-\lambda c) H \quad \sigma \lambda]$.

Property A.5. Given a matrix $\Lambda \in \mathbb{R}^{n \times m}$, a zonotope $Z=g \oplus H \mathbb{B}^{r}$, and a $H$-polytope $P=\{x \in$ $\left.\mathbb{R}^{n}:|C x-d| \leq\left[\phi_{1}, \phi_{2}, \ldots, \phi_{m}\right]^{T}\right\}$, with $C \in \mathbb{R}^{m \times n}, d \in \mathbb{R}^{m}, \phi_{i} \in \mathbb{R}_{+}(i=1,2, \ldots, m)$, define a vector $\hat{g}(\Lambda)=g+\Lambda(d-C g)$ and a matrix $\hat{H}(\Lambda)=[(I-\Lambda C) H \quad \Lambda \phi]$, with a diagonal matrix $\Lambda=\operatorname{diag}\left(\phi_{1}, \phi_{2}, \ldots, \phi_{m}\right)$. Then a family of zonotopes (parameterized by the matrix $\Lambda$ ) that contains the intersection of the zonotope $Z$ and the polytope $P$ is obtained such as $Z \cap P \subseteq \hat{Z}(\Lambda)=\hat{g} \oplus \hat{H} \mathbb{B}^{r+m}$.

Property A.6. Given a family of zonotopes denoted by $Z=g \oplus \mathbf{H B} \mathbb{B}^{m}$, where $g \in \mathbb{R}^{n}$ is a real vector 
and $\mathbf{H} \in \mathbb{R}^{n \times m}$ is an interval matrix, a zonotope inclusion $\diamond(Z)$ is defined by

$$
\diamond(Z)=g \oplus[\operatorname{mid}(\mathbf{H}) H] \mathbb{B}^{m+n},
$$

where the matrix $H$ is a diagonal matrix with

$$
H_{i i}=\sum_{j=1}^{m} \frac{\operatorname{diam}(\mathbf{H})_{i j}}{2}, i=1,2, \ldots, n,
$$

where $\operatorname{mid}(\cdot)$ and $\operatorname{diam}(\cdot)$ compute the center and diameter of interval matrices.

Property A.7. Given $Z_{k+1}=\mathbf{A} Z_{k} \oplus \mathbf{B} u_{k}$, where $\mathbf{A}$ and $\mathbf{B}$ are interval matrices and $u_{k}$ is the input at time instant $k$, if $Z_{k}$ is a zonotope with the center $g_{k}$ and segment matrix $H_{k}, Z_{k+1}$ can be bounded by a zonotope

$$
Z_{k+1}^{e}=g_{k+1} \oplus H_{k+1} \mathbb{B}^{r}
$$

with

$$
\begin{aligned}
g_{k+1} & \left.=\operatorname{mid}(\mathbf{A}) g_{k}+\operatorname{mid}(\mathbf{B})\right) u_{k}, \\
H_{k+1} & =\left[\begin{array}{lll}
J_{1} & J_{2} & J_{3}
\end{array}\right], \\
J_{1} & =\operatorname{seg}\left(\diamond\left(\mathbf{A} H_{k}\right)\right), \\
J_{2} & =\frac{\operatorname{diam}(\mathbf{A})}{2} g_{k}, \\
J_{3} & =\frac{\operatorname{diam}(\mathbf{B})}{2} u_{k},
\end{aligned}
$$

where $\operatorname{seg}(\cdot)$ computes the segment matrix of a zonotope.

\section{Appendix B. Invariant Sets}

According to (Kofman et al. 2007; Olaru et al. 2010), the linear discrete time-invariant dynamics are used to present the invariant set notions, i.e.,

$$
x_{k+1}=A_{\circ} x_{k}+B_{\circ} \delta_{k},
$$

where $A_{\circ}$ and $B_{\circ}$ are constant matrices and $A_{\circ}$ is a Schur matrix, $\delta_{k}$ belongs to $\Delta=\left\{\delta:\left|\delta-\delta^{\circ}\right| \leq \bar{\delta}\right\}$ with $\delta^{\circ}$ and $\bar{\delta}$ constant and all the matrices in (B1) have suitable dimensions.

Definition B.1. A set $X \subset \mathbb{R}^{n}$ is called an RPI set for (B1) if and only if $A_{\circ} X \oplus B_{\circ} \Delta \subseteq X$.

Definition B.2. The mRPI set of (B1) is defined as an RPI set contained in any closed RPI set and the $\mathrm{mRPI}$ set is unique and compact.

Theorem B.1. Considering (B1) and letting $A_{\circ}=V \Lambda V^{-1}$ be the Jordan decomposition of $A_{\circ}$, the set

$$
\Phi(\theta)=\left\{x \in \mathbb{R}^{n}:\left|V^{-1} x\right| \leq(I-|\Lambda|)^{-1}\left|V^{-1} B_{\circ}\right| \bar{\delta}+\theta\right\} \oplus \xi^{\circ},
$$

is RPI and attractive for the trajectories of (B1), with $\theta$ any (arbitrarily small) vector with positive components, where $\xi^{\circ}=\left(I-A_{\circ}\right)^{-1} B_{\circ} \delta^{\circ}$.

(1) For any $\theta$, the set $\Phi(\theta)$ is (positively) invariant, that is, if $x_{0} \in \Phi(\theta)$, then $x_{k} \in \Phi(\theta)$ for all $k \geq 0$.

(2) Given $\theta \in \mathbb{R}^{n}, \theta>0$, and $x_{0} \in \mathbb{R}^{n}$, there exists $k^{*} \geq 0$ such that $x_{k} \in \Phi(\theta)$ for all $k \geq k^{*}$. 
Proposition B.1. Considering the dynamics (B1), the set sequence

$$
X_{j+1}=A_{\circ} X_{j} \oplus B_{\circ} \Delta, j=0,1,2, \ldots
$$

converges to the mRPI set of the dynamics, where if $X_{0}$ is RPI, each iteration of the set sequence is an $R P I$ approximation of the mRPI set of the dynamics.

Definition B.3. Given a scalar $\epsilon>0$ and a set $\Omega \subset \mathbb{R}^{n}$, the set $\Phi \subset \mathbb{R}^{n}$ is an outer $\epsilon$-approximation of $\Omega$ if $\Omega \subseteq \Phi \subseteq \Omega \oplus \mathbb{B}_{s}^{n}(\epsilon)$ and it is an inner $\epsilon$-approximation of $\Omega$ if $\Phi \subseteq \Omega \subseteq \Phi \oplus \mathbb{B}_{s}^{n}(\epsilon)$, where $\mathbb{B}_{s}^{n}(\epsilon)=\left\{x \in \mathbb{R}^{n}:\|x\|_{s} \leq \epsilon\right\}$.

\section{Acknowledgment}

The work has been partially funded by the DGR of Generalitat de Catalunya (SAC group Ref.2009/SGR/1491), European Commission through contract i-Sense (FP7-ICT-2009-6-270428), China Scholarship Council (File No.2011629170), and the Sectoral Operational Programme Human Resources Development 2007-2013 of the Ministry of European Funds through the Financial Agreement POSDRU/159/1.5/S/132395.

\section{References}

Alamo, T., Bravo, J., and Camacho, E. (2005), "Guaranteed state estimation by zonotopes," Automatica, 41(6), 1035-1043.

Blanchini, F., and Miani, S., Set-theoretic Methods in Control, Birkhäuser Boston (2008).

Blanke, M., Kinnaert, M., Lunze, J., and Staroswiecki, M., Diagnosis and Fault-Tolerant Control, Berlin, Germany: Springer-Verlag (2006).

Blesa, J., Puig, V., Romera, J., and Saludes, J. (2011), "Fault Diagnosis of Wind Turbines using a Set-membership Approach," in Proceedings of the 18th IFAC World Congress, 28 August - 2 September, Milano, Italy.

Chai, W., and Qiao, J. (2014), "Passive robust fault detection using RBF neural modeling based on set membership identification," Engineering Applications of Artificial Intelligence, 28(0), 1 - 12.

Combastel, C. (2003), "A state bounding observer based on zonotopes," in Proceedings of the 2003 European Control Conference, Cambridge, UK.

Guerra, P., Puig, V., and Witczak, M. (2008), "Robust Fault Detection with Unknown-Input Interval Observers using Zonotopes," in Proceedings of the 17th IFAC World Congress, July, Seoul, South Korea.

Kofman, E., Haimovich, H., and Seron, M. (2007), "A systematic method to obtain ultimate bounds for perturbed systems," International Journal of Control, 80(2).

Kühn, W. (1998), "Rigorously computed orbits of dynamical systems without the wrapping effect," Computing, $61(1)$

Le, V., Stoica, C., Alamo, T., Camacho, E., and Dumur, D. (2013), "Zonotope-based Set-Membership Estimation for Multi-Output Uncertain Systems," in Proceedings of 2013 IEEE international Symposium on Intelligent Control (ISIC), Part of 2013 IEEE Multi-Conference on Systems and Control, August, Hyderabad, India.

Mazenc, F., and Bernard, O. (2011), "Interval observers for linear time-invariant systems with disturbances," $\mathrm{Au}$ tomatica, 47(1), 140 - 147.

Odgaard, P., Stoustrup, J., and Kinnaert, M. (2009), "Fault Tolerant Control of Wind Turbines-a benchmark model," in Proceedings of the 7th IFAC Symposium on Fault Detection, Supervision and Safety of Technical Process, July, Barcelona, Spain.

Olaru, S., Doná, J.D., Seron, M., and Stoican, F. (2010), "Positive invariant sets for fault tolerant multisensor control schemes," International Journal of Control, 83(12), 2622-2640.

Puig, V. (2010), "Fault diagnosis and fault tolerant control using set-membership approaches: Application to real case studies," International Journal of Applied Mathematics and Computer Science, 20(4), 619-635.

Puig, V., Quevedo, J., Escobet, T., and Stancu, A. (2003), "Passive robust fault detection using linear interval observers," in Proceedings of the 5th IFAC Symposium SAFEPROCESS-2003: Fault Detection, Supervision and Safety for Technical Processes, August, Washington, USA. 
Raïssi, T., Videau, G., and Zolghadri, A. (2010), "Interval observer design for consistency checks of nonlinear continuous-time systems," Automatica, 46(3), 518 - 527.

Seron, M., Doná, J.D., and Olaru, S. (2012), "Fault Tolerant Control Allowing Sensor Healthy-to-Faulty and Faultyto-Healthy Transitions," IEEE Transactions on Automatic Control, 57(7), 1657-1669.

Seron, M., Zhuo, X., Doná, J.D., and Martínez, J. (2008), "Multisensor switching control strategy with fault tolerance guarantees," Automatica, 44(1), 88-97.

Stoican, F., and Olaru, S., Set-Theoretic Fault Tolerant Control in Multisensor Systems, Engineering \& Materials Science. London: Wiley - ISTE (2013).

Stoican, F., Raduinea, C., and Olaru, S. (2011), "Adaptation of set theoretic methods to the fault detection of a wind turbine benchmark," in Proceedings of the 18th IFAC World Congress, 28 August-2 September, Milano, Italy.

Xu, F., Puig, V., Ocampo-Martinez, C., Stoican, F., and Olaru, S. (2014), "Improved Fault Detection and Isolation Strategy using a Bank of Interval Observers," in Proceedings of the 19th IFAC World Congress, August 24 - 29 , Cape Town, South Africa.

Xu, F., Stoican, F., Puig, V., Ocampo-Martinez, C., and Olaru, S. (2013), "Fault Detection and Isolation based on the combination of a Bank of Interval Observers and Invariant Sets," in Proceedings of the 21st Mediterranean Conference on Control and Automation, June 25 - 28, Chania, Greece. 